\title{
From ketenimines to ketenes to quinolones: two consecutive pseudopericyclic events
}

\author{
Mateo Alajarín,*a María-Mar Ortín, a Pilar Sánchez-Andrada, ${ }^{\text {a }}$ Ángel Vidal, \\ Delia Bautista $^{b}$ \\ ${ }^{a}$ Departamento de Química Orgánica, Facultad de Química, Universidad de Murcia, \\ Campus de Espinardo, 30100, Murcia, Spain \\ ${ }^{b}$ Servicio Universitario de Instrumentación Científica, Universidad de Murcia, Campus de \\ Espinardo, 30100, Murcia, Spain
}

\section{General Methods}

All melting points were determined on a Kofler hot-plate melting point apparatus and are uncorrected. Infrared (IR) spectra were recorded neat or as nujol emulsions on a Nicolet Impact 400 spectrophotometer. ${ }^{1} \mathrm{H}$ NMR and ${ }^{13} \mathrm{C}$ NMR spectra were recorded on a Bruker Avance $200\left(200 \mathrm{MHz}\right.$ and $50 \mathrm{MHz}$ for ${ }^{1} \mathrm{H}$ and ${ }^{13} \mathrm{C}$, respectively), a Bruker Avance 300 (300 $\mathrm{MHz}$ and $75 \mathrm{MHz}$ for ${ }^{1} \mathrm{H}$ and ${ }^{13} \mathrm{C}$, respectively) or on a Bruker Avance $400(400 \mathrm{MHz}$ and $100 \mathrm{MHz}$ for ${ }^{1} \mathrm{H}$ and ${ }^{13} \mathrm{C}$, respectively). The chemical shifts are expressed in ppm, relative to $\mathrm{Me}_{4} \mathrm{Si}$ at $\delta=0.00 \mathrm{ppm}$ for ${ }^{1} \mathrm{H}$, while the chemical shifts for ${ }^{13} \mathrm{C}$ are reported relative to the resonance of $\mathrm{CDCl}_{3} \delta=77.10 \mathrm{ppm}$. Mass spectra were recorded on a Hewlett-Packard 5993C spectrometer or on a VG-Autospec spectrometer. Microanalyses were performed on a Carlo Erba EA-1108 instrument.

Materials: 2-azidobenzoyl cloride $\mathbf{1 a},{ }^{1} \quad$ 2-azido-5-chlorobenzoyl chloride $\quad \mathbf{1 b},{ }^{1} \quad$ iodophenethylthiol, ${ }^{2} \alpha$-(methyl)benzylthiol, ${ }^{3}$ diphenylketene, ${ }^{4}$ and methylphenylketene ${ }^{5}$ were prepared by published procedures. 


\section{Preparation of 2-azidothiobenzoates 2}

To a solution of 2-azidobenzoyl chloride 1a or 2-azido-5-chlorobenzoyl chloride $\mathbf{1 b}$ (5 mmol) in anhydrous dichloromethane $(50 \mathrm{~mL})$ DMAP $(0.79 \mathrm{~g}, 6.5 \mathrm{mmol})$ and the corresponding thiol ( $5 \mathrm{mmol}$ ) were added. The reaction mixture was stirred at room temperature for 5 hours. Then $n$-hexane $(50 \mathrm{~mL})$ was added and the resulting solid was filtered and washed with $n$ hexane $(3 \times 15 \mathrm{~mL})$. From the filtrate the solvent was removed under reduced pressure, and the resulting material was purified by column chromatography [silica gel; hexanes/diethyl ether $4: 1(\mathrm{v} / \mathrm{v})]$.

$\boldsymbol{S}$-(4-Methylphenyl) 2-azidothiobenzoate 2a: yield $87 \%$; mp $91-92^{\circ} \mathrm{C}$ (colorless prisms, $\mathrm{Et}_{2} \mathrm{O} / n$-hexane); IR (nujol) 2132 (vs), 2097 (s), 1684 (vs), 1595 (m), 1578 (m), 1485 (s), 1305 (s), 1204 (s), 900 (s), 810 (m), 770 (m), 693 (s) cm ${ }^{-1} ;{ }^{1} \mathrm{H} \mathrm{NMR}\left(\mathrm{CDCl}_{3}, 200 \mathrm{MHz}\right) \delta 2.38$ (s, 3 H), 7.15-7.27 (m, $4 \mathrm{H}), 7.39$ (d, $2 \mathrm{H}, J=8.1 \mathrm{~Hz}), 7.51$ (td, $1 \mathrm{H}, J=7.7,1.4 \mathrm{~Hz}), 7.81$ (dd, 1 $\mathrm{H}, J=7.7,1.3 \mathrm{~Hz}) ;{ }^{13} \mathrm{C} \mathrm{NMR}\left(\mathrm{CDCl}_{3}, 50 \mathrm{MHz}\right) \delta 21.4,119.7,124.2(\mathrm{~s}), 124.6,129.6(\mathrm{~s})$, 129.7, 130.1, 133.2, 134.8, 137.9 (s), 139.9 (s), 189.7 (s); MS (EI, 70eV) m/z (rel int) 269 $\left(\mathrm{M}^{+}, 5\right), 90$ (100). Anal. Calcd for $\mathrm{C}_{14} \mathrm{H}_{11} \mathrm{~N}_{3} \mathrm{OS}$ (269.33): C, 62.43; H, 4.12; N, 15.60. Found: C, 62.47; H, 4.24; N, 15.39 .

S-(4-Methoxyphenyl) 2-azidothiobenzoate $2 \mathbf{b}$ : yield $73 \%$; $\mathrm{mp}$ 89-90 ${ }^{\circ} \mathrm{C}$ (colorless prisms, $\mathrm{Et}_{2} \mathrm{O} / n$-hexane); IR (nujol) 2129 (vs), 2092 (vs), 1684 (vs), 1593 (s), 1578 (s), 1296 (vs), 1250 (vs), 1198 (s), 1177 (s), 1105 (m), 1034 (m), 895 (s), 832 (m), 809 (w), 761 (m), 696 (m) $\mathrm{cm}^{-1} ;{ }^{1} \mathrm{H}$ NMR $\left(\mathrm{CDCl}_{3}, 200 \mathrm{MHz}\right) \delta 3.83(\mathrm{~s}, 3 \mathrm{H}), 6.97(\mathrm{~d}, 2 \mathrm{H}, J=8.8 \mathrm{~Hz}), 7.21(\mathrm{t}, 1 \mathrm{H}$, $J=7.7 \mathrm{~Hz}), 7.24(\mathrm{~d}, 1 \mathrm{H}, J=7.7 \mathrm{~Hz}), 7.42(\mathrm{~d}, 2 \mathrm{H}, J=8.8 \mathrm{~Hz}), 7.53(\mathrm{td}, 1 \mathrm{H}, J=7.7,1.4$ $\mathrm{Hz}), 7.82(\mathrm{dd}, 1 \mathrm{H}, J=7.7,1.4 \mathrm{~Hz}) ;{ }^{13} \mathrm{C} \mathrm{NMR}\left(\mathrm{CDCl}_{3}, 50 \mathrm{MHz}\right) \delta 55.4,115.0,118.4(\mathrm{~s})$, 119.7, 124.6, 129.5 (s), 129.8, 133.2, 136.4, 137.9 (s), 160.9 (s), 190.3 (s); MS (EI, 70 eV) $m / z$ (rel int) $285\left(\mathrm{M}^{+}, 39\right), 63$ (100). Anal. Calcd for $\mathrm{C}_{14} \mathrm{H}_{11} \mathrm{~N}_{3} \mathrm{O}_{2} \mathrm{~S}$ (285.32): C, 58.93; H, 3.88; N, 14.73. Found: C, 58.78; H, 3.69; N, 14.67.

S-(Pyridin-4-yl) 2-azidothiobenzoate 2c: yield 48\%; colorless oil; IR (neat) 2131 (vs), 1698 (vs), 1665 (s), 1598 (s), 1576 (vs), 1484 (vs), 1450 (s), 1410 (s), 1299 (vs), 1199 (vs), 1095 (m), 903 (vs), 818 (s), 766 (s), 714 (s), 692 (s), 651 (s) cm ${ }^{-1} ;{ }^{1} \mathrm{H}$ NMR (CDCl, $\left.400 \mathrm{MHz}\right) \delta$ $7.25(\mathrm{td}, 1 \mathrm{H}, J=7.8,1.0 \mathrm{~Hz}), 7.29(\mathrm{~d}, 1 \mathrm{H}, J=8.2 \mathrm{~Hz}), 7.47$ (dd, $2 \mathrm{H}, J=4.5,1.5 \mathrm{~Hz}), 7.59$ (ddd, $1 \mathrm{H}, J=8.2,7.5,1.5 \mathrm{~Hz}), 7.84(\mathrm{dd}, 1 \mathrm{H}, J=7.8,1.5 \mathrm{~Hz}), 8.68(\mathrm{dd}, 2 \mathrm{H}, J=4.5,1.5$ 
$\mathrm{Hz}) ;{ }^{13} \mathrm{C} \mathrm{NMR}\left(\mathrm{CDCl}_{3}, 100 \mathrm{MHz}\right) \delta 119.8,124.8,128.2,128.6$ (s), 129.9, 133.9, 138.4 (s), 138.7 (s), 150.2, 186.5 (s); MS [FAB(+)] m/z (rel int) $257\left(\mathbf{M}^{+}+1,100\right), 229\left[\left(\mathbf{M}^{+}+1\right)-\mathrm{N}_{2}\right.$, 38]. Anal. Calcd for $\mathrm{C}_{12} \mathrm{H}_{8} \mathrm{~N}_{4} \mathrm{OS}$ (256.29): C, 56.24; H, 3.15; N, 21.86. Found: C, 56.47; H, $3.24 ; \mathrm{N}, 21.65$.

S-(4-Methylphenyl) 2-azido-5-chlorothiobenzoate 2d: yield 71\%; mp 88-90 ${ }^{\circ} \mathrm{C}$ (yellow prisms, Et ${ }_{2} \mathrm{O}$ ); IR (nujol) 2143 (s), 2106 (s), 1695 (vs), 1652 (m), 1572 (w), 1321 (s), 1193 (s), 1118 (m), 931 (s), 883 (m), 819 (s), 755 (m), 723 (w), 659 (w) cm ${ }^{-1} ;{ }^{1} \mathrm{H} \mathrm{NMR} \mathrm{(CDCl}{ }_{3}$, $300 \mathrm{MHz}) \delta 2.40(\mathrm{~s}, 3 \mathrm{H}), 7.18(\mathrm{~d}, 1 \mathrm{H}, J=8.7 \mathrm{~Hz}), 7.25-7.28(\mathrm{~m}, 2 \mathrm{H}), 7.36-7.40(\mathrm{~m}, 2 \mathrm{H})$, $7.48(\mathrm{dd}, 1 \mathrm{H}, J=8.7,2.4 \mathrm{~Hz}), 7.77(\mathrm{~d}, 1 \mathrm{H}, J=2.4 \mathrm{~Hz}) ;{ }^{13} \mathrm{C} \mathrm{NMR}\left(\mathrm{CDCl}_{3}, 75 \mathrm{MHz}\right) \delta 21.5$, 121.0, 123.7 (s), 129.5, 130.2 (s), 130.3, 130.7 (s), 132.9, 134.7, 136.6 (s), 140.3 (s), 188.8 (s); MS (EI, $70 \mathrm{eV}) \mathrm{m} / \mathrm{z}$ (rel int) $305\left(\mathrm{M}^{+}+2,8\right), 303\left(\mathrm{M}^{+}, 21\right), 123$ (100). Anal. Calcd for $\mathrm{C}_{14} \mathrm{H}_{10} \mathrm{ClN}_{3} \mathrm{OS}$ (303.77): C, 55.36; H, 3.32; N, 13.83. Found: C, 55.47; H, 3.24; N, 13.98.

$S$-(4-Methoxyphenyl) 2-azido-5-chlorothiobenzoate 2e: yield 82\%; mp 83-85 $\mathrm{C}$ (yellow needles, $n$-pentane); IR (nujol) 2137 (vs), 2104 (s), 1693 (vs), 1593 (s), 1573 (m), 1308 (vs), 1255 (vs), 1196 (s), 1176 (s), 1123 (m), 1036 (m), 930 (s), 831 (vs), 758 (s) cm ${ }^{-1}$; ${ }^{1} \mathrm{H}$ NMR $\left(\mathrm{CDCl}_{3}, 200 \mathrm{MHz}\right) \delta 3.84(\mathrm{~s}, 3 \mathrm{H}), 6.95-7.01(\mathrm{~m}, 2 \mathrm{H}), 7.17(\mathrm{~d}, 1 \mathrm{H}, J=8.6 \mathrm{~Hz}), 7.37-7.43$ $(\mathrm{m}, 2 \mathrm{H}), 7.48(\mathrm{dd}, 1 \mathrm{H}, J=8.6,2.4 \mathrm{~Hz}), 7.76(\mathrm{~d}, 1 \mathrm{H}, J=2.4 \mathrm{~Hz}) ;{ }^{13} \mathrm{C} \mathrm{NMR}\left(\mathrm{CDCl}_{3}, 50\right.$ MHz) $\delta 55.4,115.1,117.8$ (s), 121.0, 129.5, 130.2 (s), 130.7 (s), 132.9, 136.4, 136.5 (s), 161.0 (s), 189.3 (s); MS (EI, $70 \mathrm{eV}) \mathrm{m} / \mathrm{z}$ (rel int) $321\left(\mathrm{M}^{+}+2,2\right), 319\left(\mathrm{M}^{+}, 7\right), 124$ (100). Anal. Calcd for $\mathrm{C}_{14} \mathrm{H}_{10} \mathrm{ClN}_{3} \mathrm{O}_{2} \mathrm{~S}$ (319.77): C, 52.59; H, 3.15; N, 13.14. Found: C, 52.47; H, $3.27 ; \mathrm{N}, 13.29$.

S-(4-Methoxybenzyl) 2-azidothiobenzoate 2f: yield 90\%; yellow oil; IR (neat) 2127 (vs), 2100 (vs), 1674 (vs), 1613 (s), 1514 (vs), 1484 (vs), 1442 (s), 1309 (vs), 1244 (vs), 1195 (vs), 1184 (s), 1100 (m), 1039 (s), 913 (vs), 769 (s), 704 (s) cm ${ }^{-1} ;{ }^{1} \mathrm{H}$ NMR (CDCl $\left.3,200 \mathrm{MHz}\right) \delta$ 3.77 (s, 3 H), 4.25 (s, 2 H), 6.81-6.88 (m, 2 H), 7.11-7.31 (m, 4 H), 7.50 (td, 1 H, J = 7.8, 1.4 $\mathrm{Hz}), 7.74(\mathrm{dd}, 1 \mathrm{H}, J=7.8,1.4 \mathrm{~Hz}) ;{ }^{13} \mathrm{C} \mathrm{NMR}\left(\mathrm{CDCl}_{3}, 50 \mathrm{MHz}\right) \delta 33.8,55.3,114.1,119.6$, 124.6, 129.0 (s), 129.5 (s), 129.8, 130.2, 133.1, 137.8 (s), 158.9 (s), 190.6 (s); MS (EI, 70 eV) $m / z$ (rel int) $299\left(\mathrm{M}^{+}, 12\right), 210$ (100). Anal. Calcd for $\mathrm{C}_{15} \mathrm{H}_{13} \mathrm{~N}_{3} \mathrm{O}_{2} \mathrm{~S}$ (299.35): C, 60.18; H, 4.38; N, 14.04. Found: C, 60.37; H, 4.23; N, 14.11. 
$S$-( $\alpha$-Methylbenzyl) 2-azidothiobenzoate 2g: yield 95\%; yellow oil; IR (neat) 2125 (vs), 1676 (vs), 1646 (s), 1592 (s), 1580 (m), 1485 (vs), 1449 (s), 1301 (vs), 1200 (vs), 1101 (m), 1051 (m), 911 (vs), 762 (vs), 700 (vs) cm ${ }^{-1} ;{ }^{1} \mathrm{H} \mathrm{NMR}\left(\mathrm{CDCl}_{3}, 400 \mathrm{MHz}\right) \delta 1.75$ (d, $3 \mathrm{H}, J=$ $7.1 \mathrm{~Hz}), 4.93(\mathrm{q}, 1 \mathrm{H}, J=7.1 \mathrm{~Hz}), 7.13(\mathrm{dt}, 1 \mathrm{H}, J=7.6,1.0 \mathrm{~Hz}), 7.19$ (dd, $1 \mathrm{H}, J=8.1,1.0$ Hz), 7.22-7.27 (m, 1 H), 7.31-7.34 (m, 2 H), 7.40-7.42 (m, 2 H), 7.47 (ddd, 1 H, J = 8.1, 7.6, $1.6 \mathrm{~Hz}), 7.71(\mathrm{dd}, 1 \mathrm{H}, J=7.8,1.6 \mathrm{~Hz}) ;{ }^{13} \mathrm{C} \mathrm{NMR}\left(\mathrm{CDCl}_{3}, 100 \mathrm{MHz}\right) \delta 22.2,43.8,119.6$, 124.5, 127.4, 127.5, 128.7, 129.6 (s), 129.8, 133.0, 137.5 (s), 142.3 (s), 190.2 (s). Anal. Calcd for $\mathrm{C}_{15} \mathrm{H}_{13} \mathrm{~N}_{3} \mathrm{OS}$ : C, 63.58; H, 4.62; N, 14.83. Found: C, 63.47; H, 4.44; N, 14.66 .

S-(4-Methoxybenzyl) 2-azido-5-chlorothiobenzoate $2 \mathrm{~h}$ : yield 80\%; yellow oil; IR (neat) 2137 (vs), 2110 (vs), 1680 (vs), 1641 (m), 1615 (m), 1514 (vs), 1476 (s), 1308 (vs), 1249 (vs), 1182 (s), 1123 (m), 1038 (m), 936 (m), 819 (s), 755 (w) cm ${ }^{-1} ;{ }^{1} \mathrm{H}$ NMR $\left(\mathrm{CDCl}_{3}, 200\right.$ MHz) $\delta 3.78$ (s, 3 H), 4.25 (s, 2 H), 6.81-6.88 (m, 2 H), 7.14 (d, 1 H, J = 8.6 Hz), 7.24-7.32 $(\mathrm{m}, 2 \mathrm{H}), 7.44(\mathrm{dd}, 1 \mathrm{H}, J=8.6,2.4 \mathrm{~Hz}), 7.70(\mathrm{~d}, 1 \mathrm{H}, J=2.4 \mathrm{~Hz}) ;{ }^{13} \mathrm{C} \mathrm{NMR}\left(\mathrm{CDCl}_{3}, 50\right.$ MHz) $\delta 34.0,55.3,114.2,120.9,128.7$ (s), 129.6, 130.1 (s), 130.2, 130.6 (s), 132.8, 136.5 (s), 159.0 (s), 189.4 (s); MS (EI, 70 eV) $m / z$ (rel int) $335\left(\mathrm{M}^{+}+2,3\right), 333\left(\mathrm{M}^{+}, 8\right), 77$ (100). Anal. Calcd for $\mathrm{C}_{15} \mathrm{H}_{12} \mathrm{ClN}_{3} \mathrm{O}_{2} \mathrm{~S}$ (333.80): C, 53.97; H, 3.62; N, 12.59. Found: C, 53.79; H, 3.46; N, 12.39 .

S-Phenethyl 2-azidothiobenzoate 2i: yield 84\%; brown oil; IR (neat) 2129 (vs), 2103 (vs), 1678 (s), 1595 (m), 1481 (s), 1447 (m), 1307 (s), 1203 (s), 912 (s), 765 (m), 700 (s) cm ${ }^{-1}$; ${ }^{1} \mathrm{H}$ NMR $\left(\mathrm{CDCl}_{3}, 200 \mathrm{MHz}\right) \delta$ 2.92-3.00 (m, $\left.2 \mathrm{H}\right), 3.24-3.32$ (m, $\left.2 \mathrm{H}\right), 7.11-7.35$ (m, $\left.7 \mathrm{H}\right), 7.48$ $(\mathrm{td}, 1 \mathrm{H}, J=8.3,1.5 \mathrm{~Hz}), 7.72(\mathrm{dd}, 1 \mathrm{H}, J=7.7,1.5 \mathrm{~Hz}) ;{ }^{13} \mathrm{C} \mathrm{NMR}\left(\mathrm{CDCl}_{3}, 50 \mathrm{MHz}\right) \delta 31.3$, 35.7, 119.6, 124.5, 126.5, 128.5, 128.6, 129.7, 130.0 (s), 133.0, 137.6 (s), 139.9 (s), 191.0 (s); MS (EI, $70 \mathrm{eV}) \mathrm{m} / z$ (rel int) $283\left(\mathrm{M}^{+}, 3\right), 105$ (100). Anal. Calcd for $\mathrm{C}_{15} \mathrm{H}_{13} \mathrm{~N}_{3} \mathrm{OS}$ (283.35): C, 63.58; H, 4.62; N, 14.83. Found: C, 63.39; H, 4.51; N, 14.74.

S-(2-iodophenethyl) 2-azidothiobenzoate 2j: yield 91\%; yellow oil; IR (neat) 2125 (vs), 2100 (vs), 1674 (vs), 1645 (s), 1596 (s), 1578 (s), 1481 (vs), 1446 (vs), 1295 (vs), 1198 (vs),

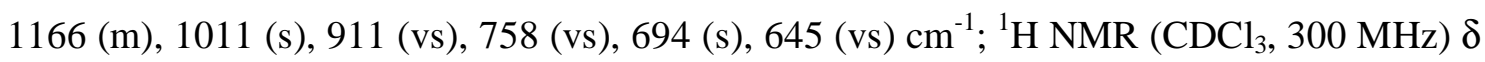
3.07-3.12 (m, 2 H), 3.26-3.31 (m, 2 H), 6.92 (ddd, 1 H, J = 8.0, 7.0, 2.2 Hz), 7.16-7.36 (m, 4 H), $7.51(\mathrm{ddd}, 1 \mathrm{H}, J=8.0,7.5,1.6 \mathrm{~Hz}), 7.75(\mathrm{dd}, 1 \mathrm{H}, J=7.8,1.6 \mathrm{~Hz}), 7.82(\mathrm{dd}, 1 \mathrm{H}, J=$ 7.8, $1.1 \mathrm{~Hz}) ;{ }^{13} \mathrm{C} \mathrm{NMR}\left(\mathrm{CDCl}_{3}, 75 \mathrm{MHz}\right) \delta 29.9,40.4,100.5$ (s), 119.6, 124.6, 128.5, 129.8, 
129.9 (s), 130.2, 133.1, 137.7 (s), 139.6, 142.6 (s), 190.9 (s). Anal. Calcd for $\mathrm{C}_{15} \mathrm{H}_{12} \mathrm{IN}_{3} \mathrm{OS}$ (409.25): C, 44.02; H, 2.95; N, 10.27. Found: C, 44.26; H, 2.87; N, 10.39.

\section{Preparation of 2-(triphenylphosphoranylideneamino)thiobenzoates 3}

To a solution of the corresponding 2-azidothiobenzoate 2 (4 mmol) in anhydrous diethyl ether $(40 \mathrm{~mL})$ triphenylphosphane $(1.05 \mathrm{~g}, 4 \mathrm{mmol})$ was added in five portions. The reaction mixture was stirred at room temperature under nitrogen for 16 hours. Then, the precipitated compounds 3 were isolated by filtration.

These compounds were used in the following reaction step without further purification. For analytical samples, compounds $\mathbf{3}$ were recrystallized from diethyl ether.

S-(4-Methylphenyl) 2-(triphenylphosphoranylideneamino)thiobenzoate 3a: yield 92\%; mp 170-172 ${ }^{\circ} \mathrm{C}$ (colorless prisms, $\mathrm{Et}_{2} \mathrm{O}$ ); IR (nujol) 1650 (s), 1587 (s), 1473 (vs), 1438 (s), 1300 (m), 1253 (m), 1183 (m), 1163 (m), 1108 (s), 1001 (m), 898 (s), 812 (w), 755 (m), 724 (s), 698 (vs), 660 (vs) cm ${ }^{-1}$; ${ }^{1} \mathrm{H} \mathrm{NMR}\left(\mathrm{CDCl}_{3}, 200 \mathrm{MHz}\right) \delta 2.37$ (s, $\left.3 \mathrm{H}\right), 6.46$ (d, $1 \mathrm{H}, J=7.5$ $\mathrm{Hz}), 6.62$ (t, $1 \mathrm{H}, J=7.5 \mathrm{~Hz}), 6.93(\mathrm{td}, 1 \mathrm{H}, J=7.5,1.8 \mathrm{~Hz}), 7.21(\mathrm{~d}, 2 \mathrm{H}, J=7.9 \mathrm{~Hz}), 7.36-$ $7.58(\mathrm{~m}, 11 \mathrm{H}), 7.70-7.90(\mathrm{~m}, 7 \mathrm{H}) ;{ }^{13} \mathrm{C} \mathrm{NMR}\left(\mathrm{CDCl}_{3}, 50 \mathrm{MHz}\right) \delta 21.4,116.8,123.0(\mathrm{~d}, J=$ $11.5 \mathrm{~Hz}), 128.2$ (s), 128.7 (d, $J=12.1 \mathrm{~Hz}), 129.6,129.7,130.3$ (d, $J=99.6 \mathrm{~Hz})(\mathrm{s}), 131.4$ (d, $J=8.2 \mathrm{~Hz})(\mathrm{s}), 132.0(\mathrm{~d}, J=2.6 \mathrm{~Hz}), 132.1,132.9$ (d, $J=9.9 \mathrm{~Hz}), 134.9,138.6$ (s), 151.9 (s), 192.1 (s); ${ }^{31} \mathrm{P}$ NMR $\left(\mathrm{CDCl}_{3}, \mathrm{H}_{3} \mathrm{PO}_{4}, 121.4 \mathrm{~Hz}\right) \delta 6.2$; MS (EI, $\left.70 \mathrm{eV}\right) \mathrm{m} / z$ (rel int) $503\left(\mathrm{M}^{+}\right.$, 2), 380 (100). Anal. Calcd for $\mathrm{C}_{32} \mathrm{H}_{26}$ NOPS (503.60): C, 76.32; H, 5.20; N, 2.78. Found: $\mathrm{C}$, 76.08; H, 5.11; N, 2.64.

S-(4-Methoxyphenyl) 2-(triphenylphosphoranylideneamino)thiobenzoate $3 \mathrm{~b}$ : yield 97\%; $\mathrm{mp} 168-170^{\circ} \mathrm{C}$ (colorless prisms, $\mathrm{Et}_{2} \mathrm{O}$ ); IR (nujol) 1635 (s), 1589 (s), 1438 (s), 1307 (m), 1250 (m), 1173 (m), 1101 (m), 1039 (w), 1012 (w), 903 (w) cm ${ }^{-1} ;{ }^{1} \mathrm{H}$ NMR $\left(\mathrm{CDCl}_{3}, 400\right.$ MHz) $\delta 3.80(\mathrm{~s}, 3 \mathrm{H}), 6.47(\mathrm{~d}, 1 \mathrm{H}, J=7.7 \mathrm{~Hz}), 6.62(\mathrm{t}, 1 \mathrm{H}, J=7.7 \mathrm{~Hz}), 6.89-6.94(\mathrm{~m}, 3 \mathrm{H})$, 7.39-7.46 (m, $8 \mathrm{H}), 7.49-7.51(\mathrm{~m}, 3 \mathrm{H}), 7.73$ (dt, $1 \mathrm{H}, J=7.9,2.1 \mathrm{~Hz}), 7.81-7.86(\mathrm{~m}, 6 \mathrm{H})$;

${ }^{13} \mathrm{C}$ NMR $\left(\mathrm{CDCl}_{3}, 100 \mathrm{MHz}\right) \delta 55.3,114.5,116.7,122.5$ (s), $123.0(\mathrm{~d}, J=11.6 \mathrm{~Hz}), 128.7$ (d, $J=12.3 \mathrm{~Hz}), 129.6$ (d, $J=2.3 \mathrm{~Hz}), 130.3$ (d, $J=100.2 \mathrm{~Hz})(\mathrm{s}), 131.3$ (d, $J=22.6 \mathrm{~Hz})(\mathrm{s})$, 131.9 (d, $J=2.7 \mathrm{~Hz}), 132.1,132.9$ (d, $J=10.1 \mathrm{~Hz}), 136.4,151.9$ (s), 160.0 (s), 192.5 (s); ${ }^{31} \mathrm{P}$ NMR $\left(\mathrm{CDCl}_{3}, \mathrm{H}_{3} \mathrm{PO}_{4}, 121.4 \mathrm{~Hz}\right) \delta$ 6.3; MS (EI, $\left.70 \mathrm{eV}\right) \mathrm{m} / z$ (rel int) 519 (M+, 3), 380 (100). 
Anal. Calcd for $\mathrm{C}_{32} \mathrm{H}_{26} \mathrm{NO}_{2} \mathrm{PS}$ (519.60): C, 73.97; H, 5.04; N, 2.70. Found: C, 73.78; H, 5.11; $\mathrm{N}, 2.54$.

S-(Pyridin-4-yl) 2-(triphenylphosphoranylideneamino)thiobenzoate 3c: yield 91\%; mp 160-162 ${ }^{\circ} \mathrm{C}$ (colorless prisms, $\mathrm{Et}_{2} \mathrm{O}$ ); IR (nujol) 1641 (vs), 1592 (s), 1574 (m), 1548 (m), 1444 (vs), 1351 (vs), 1305 (s), 1257 (s), 1166 (s), 1108 (s), 1000 (s), 906 (s), 812 (w), 757 (m), 724 (s), $710(\mathrm{~m}), 696(\mathrm{~m}) \mathrm{cm}^{-1} ;{ }^{1} \mathrm{H} \mathrm{NMR}\left(\mathrm{CDCl}_{3}, 300 \mathrm{MHz}\right) \delta 6.47(\mathrm{~d}, 1 \mathrm{H}, J=8.2 \mathrm{~Hz}), 6.64(\mathrm{td}$, $1 \mathrm{H}, J=7.9,1.0 \mathrm{~Hz}), 6.96(\mathrm{ddd}, 1 \mathrm{H}, J=8.7,7.1,1.8 \mathrm{~Hz}), 7.41(\mathrm{dd}, 2 \mathrm{H}, J=4.5,1.6 \mathrm{~Hz})$, 7.44-7.50 (m, $6 \mathrm{H}), 7.53-7.56$ (m, $3 \mathrm{H}), 7.78-7.85$ (m, $7 \mathrm{H}), 8.57$ (dd, $2 \mathrm{H}, J=4.5,1.6 \mathrm{~Hz})$; ${ }^{13} \mathrm{C} \mathrm{NMR}\left(\mathrm{CDCl}_{3}, 75 \mathrm{MHz}\right) \delta 116.9,123.1(\mathrm{~d}, J=11.9 \mathrm{~Hz}), 128.6,128.8(\mathrm{~d}, J=12.2 \mathrm{~Hz})$, $129.7(\mathrm{~d}, J=100.3 \mathrm{~Hz})(\mathrm{s}), 129.9$ (d, $J=2.7 \mathrm{~Hz}), 130.0$ (d, $J=22.5 \mathrm{~Hz})(\mathrm{s}), 132.2$ (d, $J=2.8$ $\mathrm{Hz}), 132.8$ (d, $J=10.0 \mathrm{~Hz}), 132.9,143.1$ (s), 149.6, 152.7 (s), 188.8 (s); ${ }^{31} \mathrm{P}$ NMR $\left(\mathrm{CDCl}_{3}\right.$, $\left.\mathrm{H}_{3} \mathrm{PO}_{4}, 121.4 \mathrm{~Hz}\right) \delta 8.0$; MS (EI, $\left.70 \mathrm{eV}\right) \mathrm{m} / z$ (rel int) $490\left(\mathrm{M}^{+}, 2\right), 277$ (100). Anal. Calcd for $\mathrm{C}_{30} \mathrm{H}_{23} \mathrm{~N}_{2}$ OPS (490.56): C, 73.45; H, 4.72; N, 5.71. Found: C, 73.25; H, 4.55; N, 5.64.

S-(4-Methylphenyl) 5-chloro-2-(triphenylphosphoranylideneamino)thiobenzoate 3d: yield 93\%; mp $172-174^{\circ} \mathrm{C}$ (colorless prisms, $\mathrm{Et}_{2} \mathrm{O}$ ); IR (nujol) 1647 (s), 1588 (m), 1443 (vs), 1399 (vs), 1328 (vs), 1294 (s), 1249 (m), 1163 (vs), 1110 (vs), 998 (m), 951 (s), 815 (s), 725 (vs) $\mathrm{cm}^{-1} ;{ }^{1} \mathrm{H} \mathrm{NMR}\left(\mathrm{CDCl}_{3}, 300 \mathrm{MHz}\right) \delta 2.37$ (s, $\left.3 \mathrm{H}\right), 6.37(\mathrm{dd}, 1 \mathrm{H}, J=8.8,1.3 \mathrm{~Hz}), 6.85$ (dd, $1 \mathrm{H}, J=8.8,2.8 \mathrm{~Hz}), 7.19-7.22(\mathrm{~m}, 2 \mathrm{H}), 7.35-7.37$ (m, $2 \mathrm{H}), 7.42-7.56$ (m, $9 \mathrm{H}), 7.68$ (t, $1 \mathrm{H}, J=2.6 \mathrm{~Hz}), 7.77-7.85(\mathrm{~m}, 6 \mathrm{H}) ;{ }^{13} \mathrm{C} \mathrm{NMR}\left(\mathrm{CDCl}_{3}, 75 \mathrm{MHz}\right) \delta 21.4,121.7(\mathrm{~s}), 124.0(\mathrm{~d}$, $J=11.6 \mathrm{~Hz}), 127.7(\mathrm{~s}), 128.8(\mathrm{~d}, J=12.2 \mathrm{~Hz}), 129.0(\mathrm{~d}, J=2.3 \mathrm{~Hz}), 129.7,130.0(\mathrm{~d}, J=$ $100.9 \mathrm{~Hz}$ ) (s), 131.8, 132.1 (d, $J=2.3 \mathrm{~Hz}), 132.5$ (s), 132.9 (d, $J=9.9 \mathrm{~Hz}), 134.8,138.8$ (s), $150.5(\mathrm{~d}, J=1.2 \mathrm{~Hz})(\mathrm{s}), 191.2(\mathrm{~s}) ;{ }^{31} \mathrm{P} \mathrm{NMR}\left(\mathrm{CDCl}_{3}, \mathrm{H}_{3} \mathrm{PO}_{4}, 121.4 \mathrm{~Hz}\right) \delta 7.5 ; \mathrm{MS}$ (EI, 70 eV) $m / z$ (rel int) $539\left(\mathrm{M}^{+}+2,2\right), 537\left(\mathrm{M}^{+}, 7\right), 183$ (100). Anal. Calcd for $\mathrm{C}_{32} \mathrm{H}_{25} \mathrm{ClNOPS}$ (538.05): C, 71.43; H, 4.68; N, 2.60. Found: C, 71.26; H, 4.49; N, 2.69.

S-(4-Methoxyphenyl) 5-chloro-2-(triphenylphosphoranylideneamino)thiobenzoate 3e: yield 94\%; mp 142-144 ${ }^{\circ} \mathrm{C}$ (colorless prisms, $\mathrm{Et}_{2} \mathrm{O}$ ); IR (nujol) 1639 (vs), 1591 (vs), 1445 (vs), 1401 (vs), 1340 (vs), 1293 (vs), 1251 (vs), 1171 (vs), 1116 (vs), 1021 (s), 953 (s), 837 (m), 801 (s), 721 (vs), 700 (vs), 647 (vs) cm ${ }^{-1} ;{ }^{1} \mathrm{H}$ NMR $\left(\mathrm{CDCl}_{3}, 400 \mathrm{MHz}\right) \delta 3.81$ (s, $\left.3 \mathrm{H}\right)$, $6.37(\mathrm{dd}, 1 \mathrm{H}, J=8.7,1.1 \mathrm{~Hz}), 6.85(\mathrm{dd}, 1 \mathrm{H}, J=8.7,2.7 \mathrm{~Hz}), 6.91-6.95$ (m, $2 \mathrm{H}), 7.37-7.40$ (m, 2 H), 7.44-7.48 (m, $6 \mathrm{H}), 7.52-7.56(\mathrm{~m}, 3 \mathrm{H}), 7.68$ (t, $1 \mathrm{H}, J=2.5 \mathrm{~Hz}), 7.78-7.84(\mathrm{~m}, 6$ 
$\mathrm{H}) ;{ }^{13} \mathrm{C} \mathrm{NMR}\left(\mathrm{CDCl}_{3}, 100 \mathrm{MHz}\right) \delta 55.3,114.6,121.7(\mathrm{~s}), 122.0(\mathrm{~s}), 124.0(\mathrm{~d}, J=11.4 \mathrm{~Hz})$, $128.8(\mathrm{~d}, J=12.3 \mathrm{~Hz}), 128.9(\mathrm{~d}, J=2.2 \mathrm{~Hz}), 129.8(\mathrm{~d}, J=99.7 \mathrm{~Hz})(\mathrm{s}), 131.8,132.1(\mathrm{~d}, J=$ $22.5 \mathrm{~Hz}$ ) (s), 132.2 (d, $J=2.8 \mathrm{~Hz}), 132.8$ (d, $J=22.5 \mathrm{~Hz}), 136.3,150.6$ (s), 160.2 (s), 191.7 (s); ${ }^{31} \mathrm{P} \mathrm{NMR}\left(\mathrm{CDCl}_{3}, \mathrm{H}_{3} \mathrm{PO}_{4}, 121.4 \mathrm{~Hz}\right) \delta 7.5$; MS (EI, $\left.70 \mathrm{eV}\right) \mathrm{m} / z$ (rel int) $555\left(\mathrm{M}^{+}+2,1\right)$, $533\left(\mathrm{M}^{+}, 3\right), 414$ (100). Anal. Calcd for $\mathrm{C}_{32} \mathrm{H}_{25} \mathrm{ClNO}_{2} \mathrm{PS}$ (554.05): C, 69.37; H, 4.55; N, 2.53. Found: C, 69.19; H, 4.38; N, 2.46.

S-(4-Methoxybenzyl) 2-(triphenylphosphoranylideneamino)thiobenzoate 3f: yield 90\%; $\mathrm{mp}$ 164-166 ${ }^{\circ} \mathrm{C}$ (colorless prisms, $\mathrm{Et}_{2} \mathrm{O}$ ); IR (nujol) 1617 (vs), 1592 (s), 1514 (m), 1441 (vs), 1336 (vs), 1303 (s), 1254 (s), 1180 (s), 1111 (vs), 1040 (m), 997 (s), 924 (m), 852 (m), 756 (m), $721(\mathrm{~s}), 705(\mathrm{~s}) \mathrm{cm}^{-1} ;{ }^{1} \mathrm{H} \mathrm{NMR}\left(\mathrm{CDCl}_{3}, 200 \mathrm{MHz}\right) \delta 3.76(\mathrm{~s}, 3 \mathrm{H}), 4.16(\mathrm{~s}, 2 \mathrm{H}), 6.43$ (d, $1 \mathrm{H}, J=7.4 \mathrm{~Hz}), 6.61(\mathrm{t}, 1 \mathrm{H}, J=7.4 \mathrm{~Hz}), 6.79(\mathrm{~d}, 2 \mathrm{H}, J=8.5 \mathrm{~Hz}), 6.89(\mathrm{td}, 1 \mathrm{H}, J=7.6,1.7$ $\mathrm{Hz}), 7.25(\mathrm{~d}, 2 \mathrm{H}, J=8.5 \mathrm{~Hz}), 7.32-7.53(\mathrm{~m}, 9 \mathrm{H}), 7.70-7.80(\mathrm{~m}, 7 \mathrm{H}) ;{ }^{13} \mathrm{C} \mathrm{NMR}\left(\mathrm{CDCl}_{3}, 50\right.$ MHz) $\delta 33.9,55.3,113.8,116.6,123.0(\mathrm{~d}, J=11.7 \mathrm{~Hz}), 128.6(\mathrm{~d}, J=12.3 \mathrm{~Hz}), 129.6(\mathrm{~d}, J=$ $2.6 \mathrm{~Hz}), 130.2(\mathrm{~d}, J=100.1 \mathrm{~Hz})(\mathrm{s}), 130.3,131.9(\mathrm{~d}, J=2.8 \mathrm{~Hz}), 132.0,132.1(\mathrm{~d}, J=9.7 \mathrm{~Hz})$ (s), $132.8(\mathrm{~d}, J=10.1 \mathrm{~Hz}), 151.8(\mathrm{~d}, J=1.1 \mathrm{~Hz})(\mathrm{s}), 158.4(\mathrm{~s}), 192.8(\mathrm{~d}, J=1.1 \mathrm{~Hz})(\mathrm{s}) ;{ }^{31} \mathrm{P}$ NMR $\left(\mathrm{CDCl}_{3}, \mathrm{H}_{3} \mathrm{PO}_{4}, 121.4 \mathrm{~Hz}\right) \delta$ 6.4; $\mathrm{MS}(\mathrm{EI}, 70 \mathrm{eV}) \mathrm{m} / z$ (rel int) $533\left(\mathrm{M}^{+}, 6\right), 412$ (100). Anal. Calcd for $\mathrm{C}_{33} \mathrm{H}_{28} \mathrm{NO}_{2} \mathrm{PS}$ (533.63): C, 74.28; H, 5.29; N, 2.62. Found: C, 74.08; H, 5.11; N, 2.45 .

$S$-( $\alpha$-Methylbenzyl) 2-(triphenylphosphoranylideneamino)thiobenzoate 3g: yield 97\%; $\mathrm{mp} 115-117^{\circ} \mathrm{C}$ (colorless prisms, $\mathrm{Et}_{2} \mathrm{O}$ ); IR (nujol) 1620 (vs), 1590 (vs), 1551 (m), 1380 (s), 1300 (s), 1252 (s), 1181 (s), 1158 (s), 1110 (vs), 1049 (m), 1017 (s), 916 (s), 752 (m), 722 (s), 697 (vs), 660 (s) cm ${ }^{-1} ;{ }^{1} \mathrm{H}$ NMR $\left(\mathrm{CDCl}_{3}, 400 \mathrm{MHz}\right) \delta 1.67$ (d, $\left.3 \mathrm{H}, J=7.1 \mathrm{~Hz}\right), 4.92(\mathrm{q}, 1 \mathrm{H}$, $J=7.1 \mathrm{~Hz}), 6.42(\mathrm{~d}, 1 \mathrm{H}, J=8.2 \mathrm{~Hz}), 6.61(\mathrm{t}, 1 \mathrm{H}, J=7.1 \mathrm{~Hz}), 6.89(\mathrm{ddd}, 1 \mathrm{H}, J=8.5,7.2$, $1.8 \mathrm{~Hz}), 7.20$ (tt, $1 \mathrm{H}, J=7.2,1.2 \mathrm{~Hz}), 7.25-7.29$ (m, $1 \mathrm{H}), 7.35-7.41$ (m, $9 \mathrm{H})$, 7.47-7.52 (m, $3 \mathrm{H}), 7.72-7.77(\mathrm{~m}, 7 \mathrm{H}) ;{ }^{13} \mathrm{C} \mathrm{NMR}\left(\mathrm{CDCl}_{3}, 100 \mathrm{MHz}\right) \delta 22.4,42.7,116.7,123.2(\mathrm{~d}, J=11.7$ $\mathrm{Hz}), 126.6,127.7,128.3,128.6(\mathrm{~d}, J=12.0 \mathrm{~Hz}), 129.6$ (d, $J=2.3 \mathrm{~Hz}), 130.2$ (d, $J=100.3$ Hz) (s), $131.4(\mathrm{~d}, J=22.6 \mathrm{~Hz})(\mathrm{s}), 131.8(\mathrm{~d}, J=3.1 \mathrm{~Hz}), 132.9(\mathrm{~d}, J=10.1 \mathrm{~Hz}), 133.8(\mathrm{~d}, J=$ 19.7 Hz), 144.1 (s), 151.7 (s), 192.5 (s); ${ }^{31} \mathrm{P} \mathrm{NMR}\left(\mathrm{CDCl}_{3}, \mathrm{H}_{3} \mathrm{PO}_{4}, 121.4 \mathrm{~Hz}\right) \delta$ 6.3; MS (EI, $70 \mathrm{eV}) \mathrm{m} / \mathrm{z}$ (rel int) $517\left(\mathrm{M}^{+}, 3\right), 412$ (100). Anal. Calcd for $\mathrm{C}_{33} \mathrm{H}_{28} \mathrm{NOPS}$ (517.63): C, 76.57; H, 5.45; N, 2.70. Found: C, 76.38; H, 5.31; N, 2.59 . 
S-(4-Methoxybenzyl) 5-chloro-2-(triphenylphosphoranylideneamino)thiobenzoate $3 \mathrm{~h}$ : yield 91\%; mp 140-142 ${ }^{\circ} \mathrm{C}$ (colorless prisms, $\mathrm{Et}_{2} \mathrm{O}$ ); IR (nujol) 1616 (vs), 1589 (m), 1518 (s), 1443 (vs), 1335 (vs), 1298 (s), 1245 (vs), 1167 (vs), 1113 (vs), 1014 (s), 1000 (m), 964 (m), 804 (s), 759 (s), 724 (vs), 701 (vs) cm ${ }^{-1}$; ${ }^{1} \mathrm{H}$ NMR $\left(\mathrm{CDCl}_{3}, 400 \mathrm{MHz}\right) \delta 3.78$ (s, $\left.3 \mathrm{H}\right), 4.15$ (s, $2 \mathrm{H}), 6.33(\mathrm{dd}, 1 \mathrm{H}, J=8.7,1.1 \mathrm{~Hz}), 6.79-6.84$ (m, $3 \mathrm{H}), 7.23-7.25$ (m, $2 \mathrm{H}), 7.38-7.42$ (m, 6 $\mathrm{H}), 7.49-7.53(\mathrm{~m}, 3 \mathrm{H}), 7.70-7.76(\mathrm{~m}, 7 \mathrm{H}) ;{ }^{13} \mathrm{C} \mathrm{NMR}\left(\mathrm{CDCl}_{3}, 100 \mathrm{MHz}\right) \delta 34.0,55.3,113.8$, 121.6 (s), 124.0 (d, $J=11.7 \mathrm{~Hz}), 128.7$ (d, $J=12.1 \mathrm{~Hz}), 128.9$ (d, $J=2.3 \mathrm{~Hz}), 129.7$ (d, $J=$ $111.2 \mathrm{~Hz})(\mathrm{s}), 130.2,131.6,131.7(\mathrm{~d}, J=22.6 \mathrm{~Hz})(\mathrm{s}), 132.0(\mathrm{~d}, J=2.7 \mathrm{~Hz}), 132.7(\mathrm{~d}, J=9.9$ $\mathrm{Hz}), 150.6$ (s), 158.4 (s), 191.8 (s); ${ }^{31} \mathrm{P} \mathrm{NMR}\left(\mathrm{CDCl}_{3}, \mathrm{H}_{3} \mathrm{PO}_{4}, 121.4 \mathrm{~Hz}\right) \delta 7.1$; MS (EI, 70 eV) $m / z$ (rel int) $569\left(\mathrm{M}^{+}+2,2\right), 567\left(\mathrm{M}^{+}, 3\right), 446$ (100). Anal. Calcd for $\mathrm{C}_{33} \mathrm{H}_{27} \mathrm{ClNO}_{2} \mathrm{PS}$ (568.07): C, 69.77; H, 4.79; N, 2.47. Found: C, 69.55; H, 4.81; N, 2.63.

S-Phenethyl 2-(triphenylphosphoranylideneamino)thiobenzoate 3i: yield 70\%; mp 130$132^{\circ} \mathrm{C}$ (colorless prisms, $\mathrm{Et}_{2} \mathrm{O}$ ); IR (nujol) 1670 (vs), 1593 (s), 1438 (s), 1196 (s), 1118 (vs), 1055 (w), 1026 (m), 913 (s), 763 (s), 727 (vs), 700 (vs) cm ${ }^{-1} ;{ }^{1} \mathrm{H}$ NMR (CDCl, $200 \mathrm{MHz}$ ) $\delta$ $2.93(\mathrm{t}, 2 \mathrm{H}, J=8.2 \mathrm{~Hz}), 3.21(\mathrm{t}, 2 \mathrm{H}, J=8.2 \mathrm{~Hz}), 6.44(\mathrm{~d}, 1 \mathrm{H}, J=8.2 \mathrm{~Hz}), 6.62(\mathrm{t}, 1 \mathrm{H}, J=$ $7.4 \mathrm{~Hz}), 6.90$ (t, $1 \mathrm{H}, J=7.4 \mathrm{~Hz}), 7.24$ (s ancho, $5 \mathrm{H}), 7.41-7.50(\mathrm{~m}, 9 \mathrm{H}), 7.74-7.84(\mathrm{~m}, 7 \mathrm{H})$; ${ }^{13} \mathrm{C} \mathrm{NMR}\left(\mathrm{CDCl}_{3}, 50 \mathrm{MHz}\right) \delta 31.2,35.9,116.6,123.0(\mathrm{~d}, J=11.4 \mathrm{~Hz}), 126.1,128.4,128.6$ (d, $J=12.1 \mathrm{~Hz}), 128.7,129.4,130.4$ (d, $J=100.1 \mathrm{~Hz})(\mathrm{s}), 131.5$ (s), 131.9 (d, $J=3.1 \mathrm{~Hz})$, 132.8 (d, $J=10.0 \mathrm{~Hz}), 141.2$ (s), 151.5 (s), 193.3 (s); ${ }^{31} \mathrm{P}$ NMR $\left(\mathrm{CDCl}_{3}, \mathrm{H}_{3} \mathrm{PO}_{4}, 121.4 \mathrm{~Hz}\right) \delta$ 5.4; MS (EI, $70 \mathrm{eV}) \mathrm{m} / z$ (rel int) $517\left(\mathrm{M}^{+}, 4\right), 380$ (100). Anal. Calcd for $\mathrm{C}_{33} \mathrm{H}_{28} \mathrm{NOPS}$ (517.63): C, 76.57; H, 5.45; N, 2.70. Found: C, 76.38; H, 5.31; N, 2.84.

S-(2-Iodophenethyl) 2-(triphenylphosphoranylideneamino)thiobenzoate $3 \mathrm{j}$ : yield $95 \%$; ${ }^{1} \mathrm{H} \mathrm{NMR}\left(\mathrm{CDCl}_{3}, 200 \mathrm{MHz}\right) \delta 3.01-3.08(\mathrm{~m}, 2 \mathrm{H}), 3.15-3.23(\mathrm{~m}, 2 \mathrm{H}), 6.45(\mathrm{~d}, 1 \mathrm{H}, J=8.1$ $\mathrm{Hz}), 6.63(\mathrm{t}, 1 \mathrm{H}, J=7.4 \mathrm{~Hz}), 6.82-6.95(\mathrm{~m}, 2 \mathrm{H}), 7.18-7.55(\mathrm{~m}, 12 \mathrm{H}), 7.51-7.84(\mathrm{~m}, 7 \mathrm{H})$; ${ }^{13} \mathrm{C} \mathrm{NMR}\left(\mathrm{CDCl}_{3}, 50 \mathrm{MHz}\right) \delta 29.7,40.6,100.5(\mathrm{~s}), 116.6,123.1(\mathrm{~d}, J=11.6 \mathrm{~Hz}), 128.0$, 128.4, 128.7 (d, $J=12.0 \mathrm{~Hz}), 129.4$ (d, $J=2.6 \mathrm{~Hz}), 130.1,130.3$ (d, $J=99.6 \mathrm{~Hz})(\mathrm{s}), 131.4$ $(\mathrm{d}, J=22.4 \mathrm{~Hz})(\mathrm{s}), 131.9(\mathrm{~d}, J=2.6 \mathrm{~Hz}), 132.9(\mathrm{~d}, J=9.9 \mathrm{~Hz}), 133.7(\mathrm{~d}, J=19.3 \mathrm{~Hz})$, 139.4, 143.7 (s), 151.6 (s), 193.2 (s); ${ }^{31} \mathrm{P}$ NMR $\left(\mathrm{CDCl}_{3}, \mathrm{H}_{3} \mathrm{PO}_{4}, 121.4 \mathrm{~Hz}\right) \delta 5.8$; MS (EI, 70 eV) $m / z$ (rel int) $643\left(\mathbf{M}^{+}, 14\right), 380$ (100). 


\section{Preparation of $N$-[2-(4-methylphenylthiocarbonyl)]phenyl- $C, C$-diphenylketenimine 4a}

To a solution of $S$-(4-methylphenyl) 2-(triphenylphosphoranylideneamino)thiobenzoate 3a $(0.5 \mathrm{~g}, 1 \mathrm{mmol})$ in anhydrous dichloromethane $(25 \mathrm{~mL})$ a solution of diphenylketene $(0.19 \mathrm{~g}$, $1 \mathrm{mmol})$ in the same solvent $(5 \mathrm{~mL})$ was added. After stirring at room temperature for $30 \mathrm{~min}$ the solvent was removed under reduced pressure, and the resulting material was chromatographed on a silica gel column using hexanes/diethyl ether $(4: 1, \mathrm{v} / \mathrm{v})$ as eluent.

$N$-[2-(4-methylphenylthiocarbonyl)]phenyl-C,C-diphenylketenimine 4a: yield $85 \%$; mp 125- $127^{\circ} \mathrm{C}$ (yellow needles, $\mathrm{Et}_{2} \mathrm{O} / n$-hexane); IR (nujol) 2022 (vs), 1675 (vs), 1587 (s), 1569 (m), 1494 (s), 1270 (w), 1196 (s), 1182 (s), 1162 (w), 905 (s), 811 (m), 785 (m), 766 (s), 694 (vs) $\mathrm{cm}^{-1} ;{ }^{1} \mathrm{H} \mathrm{NMR}\left(\mathrm{CDCl}_{3}, 300 \mathrm{MHz}\right) \delta 2.38(\mathrm{~s}, 3 \mathrm{H}), 7.21-7.25(\mathrm{~m}, 4 \mathrm{H})$, 7.27-7.37 (m, 12 $\mathrm{H}), 7.49(\mathrm{t}, 1 \mathrm{H}, J=8.0 \mathrm{~Hz}), 7.86(\mathrm{~d}, 1 \mathrm{H}, J=8.0 \mathrm{~Hz}) ;{ }^{13} \mathrm{C} \mathrm{NMR}\left(\mathrm{CDCl}_{3}, 75 \mathrm{MHz}\right) \delta: 21.4$, 78.1 (s), 123.6, 124.5 (s), 126.7, 127.2, 128.1, 128.9, 129.1, 130.1, 132.7 (s), 133.0, 133.5 (s), 134.8, 138.9 (s), 139.8 (s), 190.1 (s), 191.3 (s); MS (EI, 70 eV) m/z (rel int) 419 (M+, 29), 296 (100). Anal. Calcd for $\mathrm{C}_{28} \mathrm{H}_{21} \mathrm{NOS}$ (419.55): C, 80.16; H, 5.05; N, 3.34. Found: $\mathrm{C}, 80.38 ; \mathrm{H}$, $5.21 ; \mathrm{N}, 3.21$.

\section{Preparation of 2-alkyl(aryl)thio-3H-quinolin-4-ones 5}

To a solution of the corresponding 2-(triphenylphosphoranylideneamino)thiobenzoate 3 (1 $\mathrm{mmol})$ in anhydrous toluene $(15 \mathrm{~mL})$ a solution of methylphenylketene $(0.13 \mathrm{~g}, 1 \mathrm{mmol})$ or diphenylketene $(0.19 \mathrm{~g}, 1 \mathrm{mmol})$ in the same solvent $(5 \mathrm{~mL})$ was added. The reaction mixture was stirred at room temperature for $15 \mathrm{~min}$, and then was heated for 1 hour at reflux temperature. After cooling, the solvent was removed under reduced pressure, and the resulting material was chromatographed on a silica gel column using hexanes/diethyl ether $(4: 1, \mathrm{v} / \mathrm{v})$ as eluent.

Some of the 2-alkyl(aryl)thio-3H-quinolin-4-ones 5 prepared could not be obtained as crystalline solids. For these compounds, after removing the chromatographic solvents under reduced pressure the resulting solids were triturated, dried at $50^{\circ} \mathrm{C}$ under high vacuum for 24 hours, and used as such for characterization.

2-(4-Methylphenylthio)-3,3-diphenyl-3H-quinolin-4-one 5a: yield $74 \%$; mp $176-178^{\circ} \mathrm{C}$ (yellow prisms, Et ${ }_{2} \mathrm{O}$ ); IR (nujol) 1685 (vs), 1579 (s), 1561 (s), 1493 (m), 1281 (m), 1121 (m), $815(\mathrm{w}), 765(\mathrm{w}), 750(\mathrm{w}), 698(\mathrm{~m}) \mathrm{cm}^{-1} ;{ }^{1} \mathrm{H} \mathrm{NMR}\left(\mathrm{CDCl}_{3}, 400 \mathrm{MHz}\right) \delta 2.41(\mathrm{~s}, 3 \mathrm{H})$, 
$7.18(\mathrm{t}, 2 \mathrm{H}, J=6.8 \mathrm{~Hz}), 7.24(\mathrm{~d}, 2 \mathrm{H}, J=8.1 \mathrm{~Hz}), 7.31-7.36(\mathrm{~m}, 10 \mathrm{H}), 7.43(\mathrm{~d}, 2 \mathrm{H}, J=8.1$ $\mathrm{Hz}), 7.48(\mathrm{td}, 1 \mathrm{H}, J=7.6,1.5 \mathrm{~Hz}), 7.78(\mathrm{dd}, 1 \mathrm{H}, J=7.6,1.5 \mathrm{~Hz}) ;{ }^{13} \mathrm{C} \mathrm{NMR}\left(\mathrm{CDCl}_{3}, 100\right.$ MHz) $\delta 21.4,70.7$ (s), 121.8 (s), 126.2 (s), 126.5, 127.0, 127.6, 128.4, 128.5, 129.7, 130.5, 135.2, 135.7, 137.9 (s), 139.3 (s), 146.4 (s), 177.8 (s), 196.9 (s); MS (EI, 70 eV) $\mathrm{m} / z$ (rel int) $419\left(\mathrm{M}^{+}, 61\right), 296$ (100). Anal. Calcd for $\mathrm{C}_{28} \mathrm{H}_{21} \mathrm{NOS}$ (419.54): C, 80.16; H, 5.04; N, 3.34 . Found: C, 80.28; H, 5.01; N, 3.28.

3-Methyl-2-(4-methylphenylthio)-3-phenyl-3H-quinolin-4-one 5b: yield 52\%; IR (nujol) 1696 (vs), 1584 (s), 1570 (vs), 1498 (m), 1287 (m), 1225 (m), 997 (s), 811 (m), 764 (s), 708 (s) $\mathrm{cm}^{-1} ;{ }^{1} \mathrm{H}$ NMR $\left(\mathrm{CDCl}_{3}, 400 \mathrm{MHz}\right) \delta 2.01(\mathrm{~s}, 3 \mathrm{H}), 2.41(\mathrm{~s}, 3 \mathrm{H}), 7.19-7.26(\mathrm{~m}, 4 \mathrm{H}), 7.30-$ $7.34(\mathrm{~m}, 5 \mathrm{H}), 7.41$ (d, $2 \mathrm{H}, J=8.0 \mathrm{~Hz}), 7.53-7.57$ (m, $1 \mathrm{H}), 7.84(\mathrm{dd}, 1 \mathrm{H}, J=7.6,1.3 \mathrm{~Hz})$; ${ }^{13} \mathrm{C} \mathrm{NMR}\left(\mathrm{CDCl}_{3}, 100 \mathrm{MHz}\right) \delta 21.5,22.8,59.1$ (s), 120.9 (s), 125.3 (s), 126.7, 127.1, 128.1, 128.2, 129.0, 129.8, 135.3, 136.0, 138.5 (s), 139.3 (s), 146.9 (s), 180.0 (s), 197.4 (s); MS (EI, $70 \mathrm{eV}) \mathrm{m} / z$ (rel int) $357\left(\mathrm{M}^{+}, 57\right), 234$ (100). Anal. Calcd for $\mathrm{C}_{23} \mathrm{H}_{19} \mathrm{NOS}$ (357.47): C, 77.28; H, 5.36; N, 3.92. Found: C, 77.09; H, 5.21; N, 3.81.

2-(4-Methoxyphenylthio)-3,3-diphenyl-3H-quinolin-4-one 5c: yield 89\%; mp 167-168 ${ }^{\circ} \mathrm{C}$ (yellow prisms, Et ${ }_{2} \mathrm{O}$ ); IR (nujol) 1690 (vs), 1598 (s), 1579 (s), 1567 (vs), 1499 (vs), 1300 (s),

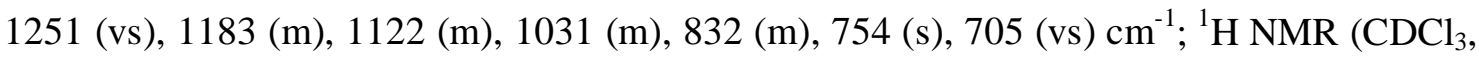
$400 \mathrm{MHz}) \delta 3.84$ (s, 3 H), 6.94-6.98 (m, 2 H), 7.13-7.19 (m, 2 H), 7.32-7.36 (m, 10 H), 7.43$7.49(\mathrm{~m}, 3 \mathrm{H}), 7.77(\mathrm{dd}, 1 \mathrm{H}, J=7.6,1.3 \mathrm{~Hz}) ;{ }^{13} \mathrm{C} \mathrm{NMR}\left(\mathrm{CDCl}_{3}, 100 \mathrm{MHz}\right) \delta 55.4,70.7$ (s), 114.6, 120.4 (s), 121.8 (s), 126.6, 127.0, 127.6, 128.4, 128.5, 130.5, 135.7, 136.9, 137.8 (s), 146.4 (s), 160.5 (s), 178.2 (s), 196.9 (s); MS (EI, $70 \mathrm{eV}) \mathrm{m} / \mathrm{z}$ (rel int) 435 (M+, 61), 295 (100). Anal. Calcd for $\mathrm{C}_{28} \mathrm{H}_{21} \mathrm{NO}_{2} \mathrm{~S}$ (435.55): C, 77.22; H, 4.86; N, 3.22. Found: C, 77.38; H, 4.71; $\mathrm{N}, 3.11$.

3,3-Diphenyl-2-(4-pyridylthio)-3H-quinolin-4-one 5d: yield 79\%; mp 144-145 ${ }^{\circ} \mathrm{C}$ (yellow prisms, $\mathrm{Et}_{2} \mathrm{O}$ ); IR (nujol) 1690 (vs), 1641 (s), 1609 (vs), 1576 (vs), 1266 (vs), 1237 (vs), 1214 (m), 1125 (vs), 1048 (vs), 818 (w), 775 (m), 756 (w), 709 (m), 661 (w) cm ${ }^{-1}$; ${ }^{1} \mathrm{H}$ NMR $\left(\mathrm{CDCl}_{3}, 400 \mathrm{MHz}\right) \delta$ 7.01-7.04 (m, $\left.2 \mathrm{H}\right), 7.33-7.41(\mathrm{~m}, 11 \mathrm{H}), 7.63(\mathrm{~d}, 1 \mathrm{H}, J=7.8 \mathrm{~Hz}), 7.68-$ $7.74(\mathrm{~m}, 3 \mathrm{H}), 7.85$ (dd, $1 \mathrm{H}, J=7.7,1.3 \mathrm{~Hz}) ;{ }^{13} \mathrm{C} \mathrm{NMR}\left(\mathrm{CDCl}_{3}, 100 \mathrm{MHz}\right) \delta 68.3$ (s), 120.7 (s), 127.7, 128.9, 129.1, 129.4, 129.56, 129.59, 129.6, 129.7, 134.6 (s), 136.6, 144.6 (s), 159.4 (s), 194.1 (s), 197.9 (s); MS (EI, $70 \mathrm{eV}$ ) m/z (rel int) $406\left(\mathrm{M}^{+}, 60\right), 296$ (100). Anal. 
Calcd for $\mathrm{C}_{26} \mathrm{H}_{18} \mathrm{~N}_{2} \mathrm{OS}$ (406.51): C, 76.82; H, 4.46; N, 6.89. Found: C, 76.68; H, 4.61; N, 6.65 .

6-Chloro-2-(4-methylphenylthio)-3,3-diphenyl-3H-quinolin-4-one $5 \mathrm{e}$ : yield $97 \%$; IR (nujol) 1696 (vs), 1577 (vs), 1557 (vs), 1296 (s), 1255 (m), 1196 (m), 1126 (s), 1079 (m), 1032 (w), 917 (w), 838 (m), 818 (m), 749 (s), 705 (vs) cm ${ }^{-1} ;{ }^{1} \mathrm{H}$ NMR (CDCl $\left.30300 \mathrm{MHz}\right) \delta$ 2.40 (s, $3 \mathrm{H}), 7.12$ (d, $1 \mathrm{H}, J=8.6 \mathrm{~Hz}), 7.22-7.24(\mathrm{~m}, 2 \mathrm{H}), 7.28-7.38$ (m, $11 \mathrm{H}), 7.40-7.43$ $(\mathrm{m}, 2 \mathrm{H}), 7.72(\mathrm{~d}, 1 \mathrm{H}, J=2.6 \mathrm{~Hz}) ;{ }^{13} \mathrm{C} \mathrm{NMR}\left(\mathrm{CDCl}_{3}, 75 \mathrm{MHz}\right) \delta 21.4,70.7(\mathrm{~s}), 122.8(\mathrm{~s})$, 125.9 (s), 126.2, 128.6, 128.7, 129.1, 129.8, 130.5, 132.4 (s), 135.2, 135.4, 137.4 (s), 139.5 (s), 144.8 (s), 178.6 (s), 195.8 (s); MS (EI, $70 \mathrm{eV}) \mathrm{m} / z$ (rel int) $455\left(\mathrm{M}^{+}+2,8\right), 453\left(\mathrm{M}^{+}, 18\right)$, 165 (100). Anal. Calcd for $\mathrm{C}_{28} \mathrm{H}_{20}$ CINOS (453.99): C, 74.08; H, 4.44; N, 3.09. Found: C, $74.28 ; \mathrm{H}, 4.61 ; \mathrm{N}, 3.01$.

6-Chloro-2-(4-methoxyphenylthio)-3,3-diphenyl-3H-quinolin-4-one 5f: yield 95\%; IR (nujol) 1695 (s), 1596 (s), 1576 (vs), 1561 (vs), 1495 (vs), 1302 (s), 1251 (vs), 1176 (m), $1130(\mathrm{~m}), 1040(\mathrm{~m}), 833(\mathrm{~m}), 705(\mathrm{~s}) \mathrm{cm}^{-1} ;{ }^{1} \mathrm{H}$ NMR $\left(\mathrm{CDCl}_{3}, 300 \mathrm{MHz}\right) \delta 3.84(\mathrm{~s}, 3 \mathrm{H}), 6.94-$ $6.97(\mathrm{~m}, 2 \mathrm{H}), 7.12(\mathrm{~d}, 1 \mathrm{H}, J=8.6 \mathrm{~Hz}), 7.27-7.45(\mathrm{~m}, 13 \mathrm{H}), 7.72(\mathrm{~d}, 1 \mathrm{H}, J=2.4 \mathrm{~Hz}) ;{ }^{13} \mathrm{C}$ NMR $\left(\mathrm{CDCl}_{3}, 75 \mathrm{MHz}\right) \delta 55.4,70.7$ (s), 114.7, 120.1 (s), 122.8 (s), 126.2, 128.6, 128.7, 129.1, 130.5, 132.4 (s), 135.4, 136.8, 137.4 (s), 144.8 (s), 160.7 (s), 179.1 (s), 195.9 (s); MS (EI, $70 \mathrm{eV}) \mathrm{m} / z$ (rel int) $471\left(\mathrm{M}^{+}+2,27\right), 469\left(\mathrm{M}^{+}, 64\right), 139$ (100). Anal. Calcd for $\mathrm{C}_{28} \mathrm{H}_{20} \mathrm{ClNO}_{2} \mathrm{~S}$ (469.99): C, 71.55; H, 4.29; N, 2.98. Found: C, 71.39; H, 4.31; N, 2.84.

6-Chloro-2-(4-methoxyphenylthio)-3-methyl-3-phenyl-3H-quinolin-4-one 5g: yield 76\%; $\mathrm{mp} 137-138^{\circ} \mathrm{C}$ (yellow prisms, $\mathrm{Et}_{2} \mathrm{O}$ ); IR (nujol) 1686 (s), 1578 (vs), 1299 (s), 1252 (vs),

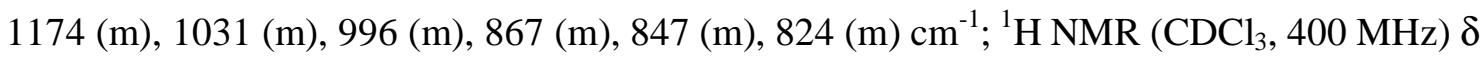
2.00 (s, $3 \mathrm{H}), 3.84$ (s, $3 \mathrm{H}), 6.93-6.97$ (m, $2 \mathrm{H}), 7.18(\mathrm{~d}, 1 \mathrm{H}, J=8.5 \mathrm{~Hz}), 7.27-7.36(\mathrm{~m}, 5 \mathrm{H})$, 7.39-7.43 (m, $2 \mathrm{H}), 7.46(\mathrm{dd}, 1 \mathrm{H}, J=8.5,2.5 \mathrm{~Hz}), 7.76(\mathrm{~d}, 1 \mathrm{H}, J=8.5 \mathrm{~Hz}) ;{ }^{13} \mathrm{C} \mathrm{NMR}$ $\left(\mathrm{CDCl}_{3}, 75 \mathrm{MHz}\right) \delta 22.6,55.4,59.0$ (s), 114.7, 119.1 (s), 121.9 (s), 126.3, 127.0, 128.3, 129.1, 129.6, 132.5 (s), 135.6, 137.0, 138.1 (s), 145.4 (s), 160.7 (s), 181.2 (s), 196.3 (s); MS (EI, $70 \mathrm{eV}) \mathrm{m} / z$ (rel int) $409\left(\mathrm{M}^{+}+2,27\right), 407\left(\mathrm{M}^{+}, 69\right), 268$ (100). Anal. Calcd for $\mathrm{C}_{23} \mathrm{H}_{18} \mathrm{ClNO}_{2} \mathrm{~S}$ (407.92): C, 67.72; H, 4.45; N, 3.43. Found: C, 67.54; H, 4.61; N, 3.21. 
2-(4-Methoxybenzylthio)-3,3-diphenyl-3H-quinolin-4-one 5 h: yield $83 \%$; mp $172-173^{\circ} \mathrm{C}$ (yellow prisms, $\mathrm{Et}_{2} \mathrm{O}$ ); IR (nujol) 1696 (s), 1604 (m), 1559 (vs), 1513 (s), 1286 (m), 1248 (vs), 1180 (m), 1125 (s), 1034 (m), 840 (w), 752 (m), 696 (s) cm ${ }^{-1} ;{ }^{1} \mathrm{H}$ NMR $\left(\mathrm{CDCl}_{3}, 400\right.$ MHz) $\delta 3.78$ (s, 3 H), 4.34 (s, 2 H), 6.81-6.85 (m, 2 H), 7.09-7.12 (m, 4 H), 7.18-7.29 (m, 7 H), 7.33-7.35 (m, $2 \mathrm{H}), 7.52$ (d, $1 \mathrm{H}, J=7.6 \mathrm{~Hz}), 7.60$ (td, $1 \mathrm{H}, J=7.6,1.5 \mathrm{~Hz}), 7.80$ (dd, 1 $\mathrm{H}, J=7.6,1.5 \mathrm{~Hz}) ;{ }^{13} \mathrm{C} \mathrm{NMR}\left(\mathrm{CDCl}_{3}, 100 \mathrm{MHz}\right) \delta 35.5,55.4,70.5$ (s), 113.8, $122.0(\mathrm{~s})$, 126.7, 126.9, 127.1, 128.3, 128.4, 129.2 (s), 130.3, 130.8, 135.9, 137.9 (s), 146.5 (s), 158.9 (s), 177.6 (s), 196.9 (s); MS (EI, $70 \mathrm{eV}) \mathrm{m} / \mathrm{z}$ (rel int) $449\left(\mathrm{M}^{+}, 18\right), 121$ (100). Anal. Calcd for $\mathrm{C}_{29} \mathrm{H}_{23} \mathrm{NO}_{2} \mathrm{~S}$ (449.57): C, 77.48; H, 5.16; N, 3.12. Found: C, 77.27; H, 5.01; N, 3.00.

3-Methyl-2-( $\alpha$-methylbenzylthio)-3-phenyl-3H-quinolin-4-one 5i: yield 58\%; IR (nujol) 1688 (vs), 1582 (vs), 1565 (vs), 1495 (s), 1290 (s), 1246 (w), 1218 (m), 1158 (m), 1034 (m),

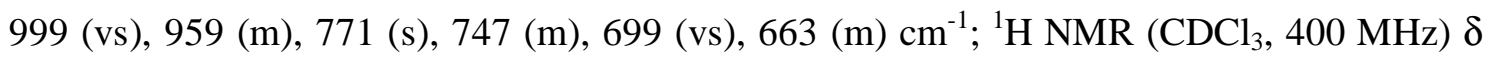
$1.71(\mathrm{~d}, 3 \mathrm{H}, J=7.2 \mathrm{~Hz}), 1.72$ (d, $3 \mathrm{H}, J=7.2 \mathrm{~Hz}), 1.76$ (s, $3 \mathrm{H}), 1.89$ (s, $3 \mathrm{H}), 5.19$ (q, $1 \mathrm{H}, J$ $=7.2 \mathrm{~Hz}), 5.20(\mathrm{q}, 1 \mathrm{H}, J=7.2 \mathrm{~Hz}), 6.92-6.95(\mathrm{~m}, 2 \mathrm{H}), 7.08-7.15(\mathrm{~m}, 3 \mathrm{H}), 7.19-7.30(\mathrm{~m}, 13$ H), 7.41-7.44 (m, 4 H), 7.52-7.57 (m, 2 H), 7.61-7.67 (m, 2 H), 7.80 (dd, 1 H, J = 7.7, 1.5 $\mathrm{Hz}), 7.85(\mathrm{dd}, 1 \mathrm{H}, J=7.7,1.5 \mathrm{~Hz}) ;{ }^{13} \mathrm{C} \mathrm{NMR}\left(\mathrm{CDCl}_{3}, 100 \mathrm{MHz}\right) \delta 21.62,21.71,22.21$, 22.84, 43.91, 43.92, 58.79 (s), 58.98 (s), 120.94 (s), 120.98 (s), 126.73, 126.75, 126.90, $126.94,127.18,127.23,127.30,127.40,127.54,127.62$, 127.78, 127.87, 128.03, 128.25, 128.46, 128.75, 128.86, 136.06, 136.14, 138.46 (s), 138.67 (s), 142.93 (s), 142.98 (s), 146.95 (s), 147.03 (s), 178.96 (s), 179.42 (s), 197.11 (s), 197.46 (s); MS (EI, $70 \mathrm{eV}$ ) m/z (rel int) 371 $\left(\mathrm{M}^{+}, 12\right), 105$ (100). Anal. Calcd for $\mathrm{C}_{24} \mathrm{H}_{21} \operatorname{NOS}$ (371.50): C, 77.59; H, 5.70; N, 3.77. Found: C, 77.32; H, 5.49; N, 3.59.

6-Chloro-2-(4-methoxybenzylthio)-3,3-diphenyl-3H-quinolin-4-one $\mathbf{5 j}$ : yield $51 \%$; IR (nujol) 1694 (s), 1574 (vs), 1561 (vs), 1513 (vs), 1303 (s), 1253 (vs), 1137 (s), 1034 (m), 836 (m), $751(\mathrm{~m}), 702(\mathrm{~s}) \mathrm{cm}^{-1}$; ${ }^{1} \mathrm{H}$ NMR $\left(\mathrm{CDCl}_{3}, 300 \mathrm{MHz}\right) \delta 3.77(\mathrm{~s}, 3 \mathrm{H}), 4.32(\mathrm{~s}, 2 \mathrm{H}), 6.81$ 6.85 (m, 2 H), 7.06-7.10 (m, 4 H), 7.20-7.34 (m, 8 H), 7.46 (d, 1 H, J = 8.4 Hz), 7.52 (dd, 1 $\mathrm{H}, J=8.4,2.4 \mathrm{~Hz}), 7.74(\mathrm{~d}, 1 \mathrm{H}, J=7.7 \mathrm{~Hz}) ;{ }^{13} \mathrm{C} \mathrm{NMR}\left(\mathrm{CDCl}_{3}, 75 \mathrm{MHz}\right) \delta 35.6,55.3,70.5$ (s), 113.8, 122.9 (s), 126.3, 128.4, 128.5, 128.6, 128.9 (s), 130.3, 130.7, 132.3 (s), 135.6, 137.5 (s), 144.9 (s), 158.5 (s), 178.4 (s), 195.7 (s); MS (EI, 70 eV) m/z (rel int) 485 (M + 2, 10), $483\left(\mathrm{M}^{+}, 27\right), 121$ (100). Anal. Calcd for $\mathrm{C}_{29} \mathrm{H}_{22} \mathrm{ClNO}_{2} \mathrm{~S}$ (484.02): C, 71.96; H, 4.58; N, 2.89. Found: C, 71.77; H, 4.41; N, 2.65. 
2-Phenethylthio-3,3-diphenyl-3H-quinolin-4-one 5k: yield $85 \%$; mp $138-140^{\circ} \mathrm{C}$ (colorless prisms, Et ${ }_{2} \mathrm{O}$ ); IR (nujol) 1689 (s), 1599 (m), 1580 (m), 1558 (vs), 1497 (s), 1382 (m), 1289 (m), 1135 (s), 701 (s), 648 (s) cm ${ }^{-1}$; ${ }^{1} \mathrm{H}$ NMR ( $\left.\mathrm{CDCl}_{3}, 400 \mathrm{MHz}\right) \delta$ 3.00-3.04 (m, $\left.2 \mathrm{H}\right), 3.37-$ 3.41 (m, 2 H), 7.17-7.23 (m, 6 H), 7.27-7.33 (m, 10 H), 7.49 (d, 1 H, J = 7.9 Hz), 7.59 (td, 1 $\mathrm{H}, J=7.6,1.3 \mathrm{~Hz}), 7.81(\mathrm{~d}, 1 \mathrm{H}, J=7.6 \mathrm{~Hz}) ;{ }^{13} \mathrm{C} \mathrm{NMR}\left(\mathrm{CDCl}_{3}, 100 \mathrm{MHz}\right) \delta 32.6,35.3,70.8$ (s), 121.9 (s), 126.5, 126.7, 126.8, 127.2, 128.3, 128.4, 128.6, 128.7, 130.4, 135.9, 138.2 (s), 140.6 (s), 146.5 (s), 178.0 (s), 196.9 (s); MS (EI, 70 eV) m/z (rel int) 433 (M+, 6), 329 (100). Anal. Calcd for $\mathrm{C}_{29} \mathrm{H}_{23} \mathrm{NOS}$ (433.57): C, 80.34; H, 5.35; N, 3.23. Found: C, 80.16; H, 5.25; N, 3.07.

2-(2-Iodophenethylthio)-3,3-diphenyl-3H-quinolin-4-one 5l: yield 90\%; mp 131-133 ${ }^{\circ} \mathrm{C}$ (yellow prisms, $\mathrm{Et}_{2} \mathrm{O}$ ); IR (nujol) 1690 (vs), 1602 (m), 1580 (vs), 1567 (vs), 1290 (m), 1124 (s), 1017 (w), 783 (m), 772 (m), 755 (s), 746 (s), 702 (s), 661 (m), 639 (s) cm ${ }^{-1}$; ${ }^{1} \mathrm{H}$ NMR $\left(\mathrm{CDCl}_{3}, 300 \mathrm{MHz}\right) \delta 3.11-3.18(\mathrm{~m}, 2 \mathrm{H}), 3.36-3.49(\mathrm{~m}, 2 \mathrm{H}), 6.86-6.97$ (m, $\left.1 \mathrm{H}\right)$, 7.16-7.33 $(\mathrm{m}, 13 \mathrm{H}), 7.51-7.65(\mathrm{~m}, 2 \mathrm{H}), 7.81(\mathrm{~d}, 2 \mathrm{H}, J=7.8 \mathrm{~Hz}) ;{ }^{13} \mathrm{C} \mathrm{NMR}\left(\mathrm{CDCl}_{3}, 75 \mathrm{MHz}\right) \delta 31.0$, 40.2, 70.8 (s), 100.5 (s), 121.8 (s), 126.7, 126.9, 127.3, 128.3, 128.4, 128.5, 130.1, 130.4, 135.9, 138.1 (s), 139.6, 143.1 (s), 146.5 (s), 177.7 (s), 196.9 (s); MS (EI, 70 eV) m/z (rel int) 559 ( $\left.\mathrm{M}^{+}, 33\right), 329$ (100). Anal. Calcd for $\mathrm{C}_{29} \mathrm{H}_{22} \mathrm{INOS}$ (559.47): C, 62.26; H, 3.96; N, 2.50 . Found: C, 62.08; H, 3.71; N, 2.34.

\section{Preparation of bis(azides) 6}

To a solution of 2-azidobenzoyl chloride $1 \mathrm{a}(0.91 \mathrm{~g}, 5 \mathrm{mmol})$ in anhydrous dichloromethane (50 mL) DMAP (0.79 g, $6.5 \mathrm{mmol})$ and the corresponding bis(thiol) (2.5 mmol) were added. The reaction mixture was stirred at room temperature for 5 hours. Then $n$-hexane $(50 \mathrm{~mL})$ was added and the resulting solid was filtered and washed with $n$-hexane $(3 \times 15 \mathrm{~mL})$. From the filtrate the solvent was removed under reduced pressure, and the resulting material was purified by column chromatography [silica gel; hexanes/diethyl ether 4:1 (v/v)].

Bis(azide) 6a $\left(\mathrm{X}=\mathrm{CH}_{2} \mathrm{CH}_{2} \mathrm{CH}_{2}\right)$ : yield 58\%; yellow oil; IR (neat) 2129 (vs), 2097 (vs), 1676 (vs), 1595 (s), 1576 (m), 1483 (vs), 1443 (s), 1306 (vs), 1103 (w), 914 (vs), 765 (vs), 698 (s) $\mathrm{cm}^{-1} ;{ }^{1} \mathrm{H} \mathrm{NMR}\left(\mathrm{CDCl}_{3}, 400 \mathrm{MHz}\right) \delta 2.08$ (quint, $2 \mathrm{H}, J=7.1 \mathrm{~Hz}$ ), $3.17(\mathrm{t}, 4 \mathrm{H}, J=7.1 \mathrm{~Hz}$ ), $7.18(\mathrm{t}, 2 \mathrm{H}, J=7.6 \mathrm{~Hz}), 7.22$ (d, $2 \mathrm{H}, J=8.1 \mathrm{~Hz}), 7.51$ (ddd, $2 \mathrm{H}, J=8.1,7.6,1.5 \mathrm{~Hz}), 7.76$ $(\mathrm{dd}, 2 \mathrm{H}, J=7.8,1.5 \mathrm{~Hz}) ;{ }^{13} \mathrm{C} \mathrm{NMR}\left(\mathrm{CDCl}_{3}, 100 \mathrm{MHz}\right) \delta 28.7,29.2,119.6,124.6,129.7$, 
129.8 (s), 133.0, 137.6 (s), 190.8 (s); MS [FAB(+)] $m / z$ (rel int) $399\left(\mathrm{M}^{+}+1,10\right)$. Anal. Calcd for $\mathrm{C}_{17} \mathrm{H}_{14} \mathrm{~N}_{6} \mathrm{O}_{2} \mathrm{~S}_{2}$ (398.47): C, 51.24; H, 3.54; N, 21.09. Found: C, 51.09; H, 3.45; N, 20.98 .

Bis(azide) 6b $\left(\mathrm{X}=1,2-\mathrm{C}_{6} \mathrm{H}_{4}\right)$ : yield 50\%; mp 106-108 ${ }^{\circ} \mathrm{C}$ (yellow prisms, $\mathrm{Et}_{2} \mathrm{O}$ ); IR (nujol) 2134 (vs), 2094 (vs), 1696 (vs), 1584 (m), 1311 (s), 1207 (s), 1173 (m), 910 (s), 753 (s) cm cm $^{-1}$ ${ }^{1} \mathrm{H} \mathrm{NMR}\left(\mathrm{CDCl}_{3}, 400 \mathrm{MHz}\right) \delta 7.19(\mathrm{t}, 2 \mathrm{H}, J=7.6 \mathrm{~Hz}), 7.24(\mathrm{~d}, 2 \mathrm{H}, J=8.4 \mathrm{~Hz}), 7.51-7.55$ $(\mathrm{m}, 4 \mathrm{H}), 7.73-7.76(\mathrm{~m}, 2 \mathrm{H}), 7.84(\mathrm{dd}, 2 \mathrm{H}, J=7.8,1.4 \mathrm{~Hz}) ;{ }^{13} \mathrm{C} \mathrm{NMR}\left(\mathrm{CDCl}_{3}, 100 \mathrm{MHz}\right) \delta$ 119.7, 124.7, $129.2(\mathrm{~s}), 130.0,130.7,133.4,137.1,138.2(\mathrm{~s}), 188.2(\mathrm{~s}) ; \mathrm{MS}[\mathrm{FAB}(+)] \mathrm{m} / \mathrm{z}(\mathrm{rel}$ int) $433\left(\mathrm{M}^{+}+1,9\right)$. Anal. Calcd for $\mathrm{C}_{20} \mathrm{H}_{12} \mathrm{~N}_{6} \mathrm{O}_{2} \mathrm{~S}_{2}$ (432.49): C, 55.54; H, 2.80; N, 19.43. Found: C, 55.36; H, 2.68; N, 19.27 .

Bis(azida) 6c $\left(\mathrm{X}=1,3-\mathrm{C}_{6} \mathrm{H}_{4}\right)$ : yield $76 \%$; mp 103-105 ${ }^{\circ} \mathrm{C}$ (colorless prisms, $\mathrm{Et}_{2} \mathrm{O}$ ); IR (nujol) 2124 (vs), 1683 (vs), 1595 (m), 1576 (m), 1480 (s), 1293 (s), 905 (s), 764 (m), 693 (s) cm cm $^{-1}$ ${ }^{1} \mathrm{H} \mathrm{NMR}\left(\mathrm{CDCl}_{3}, 400 \mathrm{MHz}\right) \delta 7.22(\mathrm{t}, 2 \mathrm{H}, J=7.7 \mathrm{~Hz}), 7.26(\mathrm{~d}, 2 \mathrm{H}, J=8.0 \mathrm{~Hz}), 7.51-7.57$ $(\mathrm{m}, 3 \mathrm{H}), 7.59-7.61(\mathrm{~m}, 2 \mathrm{H}), 7.69$ (s, $1 \mathrm{H}), 7.84(\mathrm{dd}, 2 \mathrm{H}, J=7.8,1.2 \mathrm{~Hz}) ;{ }^{13} \mathrm{C} \mathrm{NMR}\left(\mathrm{CDCl}_{3}\right.$, $100 \mathrm{MHz}) \delta 119.7,124.7,129.0$ (s), 129.1 (s), 129.8, 129.9, 133.5, 136.0, 138.1 (s), 140.4, 188.6 (s); MS $[\mathrm{FAB}(+)] \mathrm{m} / \mathrm{z}$ (rel int) $433\left(\mathrm{M}^{+}+1\right.$, 11). Anal. Calcd for $\mathrm{C}_{20} \mathrm{H}_{12} \mathrm{~N}_{6} \mathrm{O}_{2} \mathrm{~S}_{2}$ (432.49): C, 55.54; H, 2.80; N, 19.43. Found: C, 55.28; H, 2.71; N, 19.27.

\section{Preparation of bis(triphenyliminophosphoranes) 7}

To a solution of the corresponding bis(azide) $6(2 \mathrm{mmol})$ in anhydrous diethyl ether $(40 \mathrm{~mL})$ triphenylphosphane $(1.05 \mathrm{~g}, 4 \mathrm{mmol})$ was added in five portions. The reaction mixture was stirred at room temperature under nitrogen for 16 hours. Then, the precipitated compounds 7 were isolated by filtration.

These compounds were used in the following reaction step without further purification. For analytical samples, compounds 7 were recrystallized from benzene/ $n$-hexane.

Bis(triphenyliminophosphorane) 7a $\left(\mathrm{X}=\mathrm{CH}_{2} \mathrm{CH}_{2} \mathrm{CH}_{2}\right)$ : yield $78 \%$; mp $172-173^{\circ} \mathrm{C}$ (colorless prisms, benzene/n-hexane); IR (nujol) 1635 (vs), 1591 (s), 1438 (vs), 1299 (m), 1261 (m), 1187 (m), 1160 (m), 1116 (s), 1013 (m), 914 (m), 754 (m), 725 (s), 698 (s), 663 (m) $\mathrm{cm}^{-1} ;{ }^{1} \mathrm{H} \mathrm{NMR}\left(\mathrm{CDCl}_{3}, 200 \mathrm{MHz}\right) \delta 1.99$ (quint, $2 \mathrm{H}, J=7.1 \mathrm{~Hz}$ ), $3.05(\mathrm{t}, 4 \mathrm{H}, J=7.1$ $\mathrm{Hz}), 6.44(\mathrm{~d}, 2 \mathrm{H}, J=8.2 \mathrm{~Hz}), 6.62(\mathrm{t}, 2 \mathrm{H}, J=7.5 \mathrm{~Hz}), 6.90(\mathrm{ddd}, 2 \mathrm{H}, J=8.4,7.5,1.8 \mathrm{~Hz})$, 
7.38-7.52 (m, $18 \mathrm{H})$, 7.69-7.85 (m, $14 \mathrm{H}) ;{ }^{13} \mathrm{C} \mathrm{NMR}\left(\mathrm{CDCl}_{3}, 50 \mathrm{MHz}\right) \delta 29.2,116.6,123.1$ $(\mathrm{d}, J=11.4 \mathrm{~Hz}), 128.7(\mathrm{~d}, J=12.1 \mathrm{~Hz}), 129.4(\mathrm{~d}, J=2.3 \mathrm{~Hz}), 130.4(\mathrm{~d}, J=100.2 \mathrm{~Hz})(\mathrm{s})$, 131.7, 131.8 (d, $J=22.1 \mathrm{~Hz})(\mathrm{s}), 131.9$ (d, $J=2.5 \mathrm{~Hz}), 132.9$ (d, $J=10.0 \mathrm{~Hz}), 151.5$ (s), 193.4 (s); ${ }^{31} \mathrm{P} \mathrm{NMR}\left(\mathrm{CDCl}_{3}, \mathrm{H}_{3} \mathrm{PO}_{4}, 121.4 \mathrm{~Hz}\right) \delta 5.4 ; \mathrm{MS}[\mathrm{FAB}(+)] \mathrm{m} / z$ (rel int) $867\left(\mathrm{M}^{+}+1\right.$, 59). Anal. Calcd for $\mathrm{C}_{53} \mathrm{H}_{44} \mathrm{~N}_{2} \mathrm{O}_{2} \mathrm{P}_{2} \mathrm{~S}_{2}$ (867.02): C, 73.42; H, 5.11; N, 3.23. Found: C, 73.24; H, 5.01; N, 3.09.

Bis(triphenyliminophosphorane) $7 \mathbf{b}\left(\mathrm{X}=1,2-\mathrm{C}_{6} \mathrm{H}_{4}\right)$ : yield $90 \%$; mp $114-116^{\circ} \mathrm{C}$ (yellow prisms, benzene/n-hexane); IR (nujol) 1685 (vs), 1629 (vs), 1590 (vs), 1551 (m), 1300 (s), 1253 (s), 1185 (s), 1158 (s), 1111 (vs), 1053 (w), 1012 (w), 999 (w), 898 (m), 750 (m), 719 (s), 694 (vs) cm ${ }^{-1} ;{ }^{1} \mathrm{H} \mathrm{NMR}\left(\mathrm{CDCl}_{3}, 400 \mathrm{MHz}\right) \delta 6.42(\mathrm{~d}, 2 \mathrm{H}, J=8.2 \mathrm{~Hz}), 6.48(\mathrm{t}, 2 \mathrm{H}, J=$ $7.1 \mathrm{~Hz}), 6.87$ (ddd, $2 \mathrm{H}, J=8.5,7.2,1.8 \mathrm{~Hz}), 7.29-7.33(\mathrm{~m}, 12 \mathrm{H}), 7.36-7.40$ (m, $8 \mathrm{H}), 7.59$ $(\mathrm{dt}, 2 \mathrm{H}, J=7.9,2.1 \mathrm{~Hz}), 7.66-7.69(\mathrm{~m}, 2 \mathrm{H}), 7.75-7.80(\mathrm{~m}, 12 \mathrm{H}) ;{ }^{13} \mathrm{C} \mathrm{NMR}\left(\mathrm{CDCl}_{3}, 100\right.$ MHz) $\delta 116.5,122.8(\mathrm{~d}, J=12.1 \mathrm{~Hz}), 128.7(\mathrm{~d}, J=12.1 \mathrm{~Hz}), 129.1,129.9$ (d, $J=2.3 \mathrm{~Hz})$, $130.1(\mathrm{~d}, J=99.8 \mathrm{~Hz})(\mathrm{s}), 131.2(\mathrm{~d}, J=22.6 \mathrm{~Hz})(\mathrm{s}), 131.8(\mathrm{~d}, J=2.7 \mathrm{~Hz}), 131.9,132.9$ (d, $J$ $=9.9 \mathrm{~Hz}), 136.8,137.0$ (s), 152.2 (s), 191.1 (s); ${ }^{31} \mathrm{P} \mathrm{NMR}\left(\mathrm{CDCl}_{3}, \mathrm{H}_{3} \mathrm{PO}_{4}, 121.4 \mathrm{~Hz}\right) \delta 6.6$; MS $[\mathrm{FAB}(+)] m / z$ (rel int) $901\left(\mathrm{M}^{+}+1,6\right)$. Anal. Calcd for $\mathrm{C}_{56} \mathrm{H}_{42} \mathrm{~N}_{2} \mathrm{O}_{2} \mathrm{P}_{2} \mathrm{~S}_{2}$ (901.04): $\mathrm{C}$, 74.65; H, 4.70; N, 3.11. Found: C, 74.43; H, 4.44; N, 2.99.

Bis(triphenyliminophosphorane) 7c $\left(\mathrm{X}=1,3-\mathrm{C}_{6} \mathrm{H}_{4}\right)$ : yield $94 \%$; $\mathrm{mp} 228-230^{\circ} \mathrm{C}$ (colorless prisms, benzene/n-hexane); IR (nujol) 1643 (vs), 1593 (s), 1443 (vs), 1300 (s), 1263 (s), 1118 (s), 1165 (s), 1111 (vs), 1055 (m), 1012 (s), 908 (m), 787 (m), 749 (s), 724 (s), 699 (s), 658 (s) $\mathrm{cm}^{-1} ;{ }^{1} \mathrm{H} \mathrm{NMR}\left(\mathrm{CDCl}_{3}, 300 \mathrm{MHz}\right) \delta 6.47(\mathrm{~d}, 2 \mathrm{H}, J=8.2 \mathrm{~Hz}), 6.63(\mathrm{td}, 2 \mathrm{H}, J=7.5,1.0$ $\mathrm{Hz}), 6.93$ (ddd, $2 \mathrm{H}, J=8.7,7.1,1.8 \mathrm{~Hz}), 7.42-7.54(\mathrm{~m}, 21 \mathrm{H}), 7.60$ (t, $1 \mathrm{H}, J=1.6 \mathrm{~Hz}), 7.74$ $(\mathrm{dt}, 2 \mathrm{H}, J=8.0,2.0 \mathrm{~Hz}), 7.79-7.87(\mathrm{~m}, 12 \mathrm{H}) ;{ }^{13} \mathrm{C} \mathrm{NMR}\left(\mathrm{CDCl}_{3}, 75 \mathrm{MHz}\right) \delta 116.8,123.1(\mathrm{~d}$, $J=11.6 \mathrm{~Hz}), 128.8(\mathrm{~d}, J=12.2 \mathrm{~Hz}), 128.9,129.7$ (d, $J=2.2 \mathrm{~Hz}), 130.2(\mathrm{~d}, J=100.3 \mathrm{~Hz})(\mathrm{s})$, $131.3(\mathrm{~d}, J=22.3 \mathrm{~Hz})(\mathrm{s}), 132.0(\mathrm{~d}, J=2.7 \mathrm{~Hz}), 132.2(\mathrm{~d}, J=2.2 \mathrm{~Hz}), 132.9(\mathrm{~d}, J=10.0 \mathrm{~Hz})$, 135.3, 140.6, 152.1 (s), 191.5 (s); ${ }^{31} \mathrm{P}$ NMR $\left(\mathrm{CDCl}_{3}, \mathrm{H}_{3} \mathrm{PO}_{4}, 121.4 \mathrm{~Hz}\right) \delta 6.8 ; \mathrm{MS}[\mathrm{FAB}(+)]$ $m / z$ (rel int) $901\left(\mathrm{M}^{+}+1,5\right)$. Anal. Calcd for $\mathrm{C}_{56} \mathrm{H}_{42} \mathrm{~N}_{2} \mathrm{O}_{2} \mathrm{P}_{2} \mathrm{~S}_{2}$ (901.04): C, 74.65; H, 4.70; N, 3.11. Found: C, 74.44; H, 4.52; N, 3.00. 


\section{Preparation of bis( $3 H$-quinolin-4-ones) 8}

To a solution of the corresponding bis(triphenyliminophosphorane $) 7(0.5 \mathrm{mmol})$ in anhydrous toluene $(15 \mathrm{~mL})$ a solution of methylphenylketene $(0.13 \mathrm{~g}, 1 \mathrm{mmol})$ or diphenylketene $(0.19 \mathrm{~g}, 1 \mathrm{mmol})$ in the same solvent $(5 \mathrm{~mL})$ was added. The reaction mixture was stirred at room temperature for $15 \mathrm{~min}$, and then was heated for 1 hour at reflux temperature. After cooling, the solvent was removed under reduced pressure, and the resulting material was chromatographed on a silica gel column using hexanes/diethyl ether $(4: 1, \mathrm{v} / \mathrm{v})$ as eluent.

Some of the bis( $3 H$-quinolin-4-ones) 8 prepared could not be obtained as crystalline solids. For these compounds, after removing the chromatographic solvents under reduced pressure the resulting solids were triturated, dried at $50^{\circ} \mathrm{C}$ under high vacuum for 24 hours, and used as such for characterization.

Bis(3H-quinolin-4-one) 8a: yield 89\%; mp 150-152 ${ }^{\circ} \mathrm{C}$ (yellow prisms, $\mathrm{Et}_{2} \mathrm{O}$ ); IR (nujol) 1691 (vs), 1599 (s), 1579 (s), 1559 (vs), 1281 (s), 1122 (s), 763 (m), 751 (m), 705 (s) cm'${ }^{1} \mathrm{H} \mathrm{NMR}\left(\mathrm{CDCl}_{3}, 200 \mathrm{MHz}\right) \delta 2.19$ (quint, $2 \mathrm{H}, J=7.1 \mathrm{~Hz}$ ), $3.28(\mathrm{t}, 4 \mathrm{H}, J=7.1 \mathrm{~Hz}$ ), 7.15$7.36(\mathrm{~m}, 24 \mathrm{H}), 7.53(\mathrm{td}, 2 \mathrm{H}, J=7.8,1.2 \mathrm{~Hz}), 7.80(\mathrm{dd}, 2 \mathrm{H}, J=7.8,1.0 \mathrm{~Hz}) ;{ }^{13} \mathrm{C} \mathrm{NMR}$ $\left(\mathrm{CDCl}_{3}, 50 \mathrm{MHz}\right) \delta 27.9,30.5,70.8$ (s), $121.8(\mathrm{~s}), 126.6,126.9,127.3,128.3,128.4,130.3$, 135.8, 138.1 (s), 146.4 (s), 177.9 (s), 196.9 (s); MS [FAB(+)] m/z (rel int) $699\left(\mathrm{M}^{+}+1,60\right)$. Anal. Calcd for $\mathrm{C}_{45} \mathrm{H}_{34} \mathrm{~N}_{2} \mathrm{O}_{2} \mathrm{~S}_{2}$ (698.91): C, 77.33; H, 4.90; N, 4.01. Found: C, 77.18; H, 4.71; N, 4.27.

Bis(3H-quinolin-4-one) 8b: yield 61\%; IR (nujol) 1698 (vs), 1561 (vs), 1475 (vs), 1377 (m), 1291 (vs), 1006 (vs), 962 (s), 767 (vs), 700 (vs) cm ${ }^{-1} ;{ }^{1} \mathrm{H} \mathrm{NMR}\left(\mathrm{CDCl}_{3}, 400 \mathrm{MHz}\right.$ ) $\delta 1.90$ (s, $6 \mathrm{H}), 2.13$ (quint, $2 \mathrm{H}, J=7.0 \mathrm{~Hz}$ ), 3.22-3.34 (m, $4 \mathrm{H}), 7.22-7.29(\mathrm{~m}, 12 \mathrm{H}), 7.44$ (d, $2 \mathrm{H}, J=$ $7.9 \mathrm{~Hz}), 7.61(\mathrm{t}, 2 \mathrm{H}, J=7.9 \mathrm{~Hz}), 7.87(\mathrm{~d}, 2 \mathrm{H}, J=7.7 \mathrm{~Hz}) ;{ }^{13} \mathrm{C} \mathrm{NMR}\left(\mathrm{CDCl}_{3}, 100 \mathrm{MHz}\right) \delta$ 23.1, 28.3, 29.3, 59.1 (s), 120.9 (s), 126.8, 126.9, 127.0, 127.6, 128.0, 128.9, 136.1, 138.8 (s), 146.9 (s), 179.9 (s), 197.4 (s); MS [FAB(+)] m/z (rel int) $575\left(\mathrm{M}^{+}+1\right.$, 76). Anal. Calcd for $\mathrm{C}_{35} \mathrm{H}_{30} \mathrm{~N}_{2} \mathrm{O}_{2} \mathrm{~S}_{2}$ (574.77): C, 73.14; H, 5.26; N, 4.87. Found: C, 73.01; H, 5.08; N, 4.67.

Bis(3H-quinolin-4-one) 8c: yield 64\%; mp 218-220 ${ }^{\circ} \mathrm{C}$ (yellow prisms, $\mathrm{Et}_{2} \mathrm{O}$ ); IR (nujol) 1687 (vs), 1584 (vs), 1560 (vs), 1493 (m), 1286 (s), 1124 (s), 1042 (m), 922 (w), 906 (w),

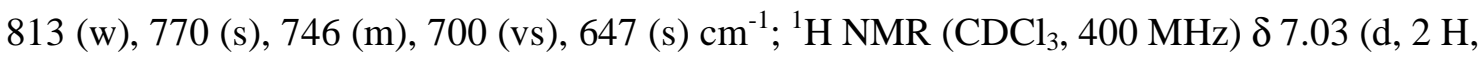


$J=8.0 \mathrm{~Hz}), 7.11(\mathrm{t}, 2 \mathrm{H}, J=7.4 \mathrm{~Hz}), 7.16-7.27(\mathrm{~m}, 20 \mathrm{H}), 7.34(\mathrm{td}, 2 \mathrm{H}, J=7.7,1.4 \mathrm{~Hz})$, 7.47-7.49 (m, $2 \mathrm{H}), 7.74-7.77(\mathrm{~m}, 4 \mathrm{H}) ;{ }^{13} \mathrm{C} \mathrm{NMR}\left(\mathrm{CDCl}_{3}, 100 \mathrm{MHz}\right) \delta 70.6(\mathrm{~s}), 121.7(\mathrm{~s})$, 126.4, 126.9, 127.6, 128.3, 128.4, 129.5, 130.3, 135.4 (s), 135.6, 137.0, 137.8 (s), 146.1 (s), 176.6 (s), 196.7 (s); MS $[\mathrm{FAB}(+)] \mathrm{m} / z$ (rel int) $733\left(\mathrm{M}^{+}+1\right.$, 76). Anal. Calcd for $\mathrm{C}_{48} \mathrm{H}_{32} \mathrm{~N}_{2} \mathrm{O}_{2} \mathrm{~S}_{2}$ (732.93): C, 78.66; H, 4.40; N, 3.82. Found: C, 78.46; H, 4.61; N, 3.65.

Bis(3H-quinolin-4-one) 8d: yield 63\%; mp 181-183 ${ }^{\circ} \mathrm{C}$ (yellow prisms, $\mathrm{Et}_{2} \mathrm{O}$ ); IR (nujol) 1690 (vs), 1585 (vs), 1568 (vs), 1495 (m), 1285 (s), 1214 (w), 1158 (w), 994 (s), 961 (w), $767(\mathrm{~m}), 750(\mathrm{~m}), 703$ (s) cm ${ }^{-1} ;{ }^{1} \mathrm{H}$ NMR $\left(\mathrm{CDCl}_{3}, 300 \mathrm{MHz}\right) \delta 1.80(\mathrm{~s}, 3 \mathrm{H}), 1.92(\mathrm{~s}, 3 \mathrm{H})$, 7.12-7.28 (m, 14 H), 7.43-7.52 (m, 4 H), 7.12-7.44 (m, 2 H), 7.82 (dt, $2 \mathrm{H}, J=7.7,1.4$ Hz); ${ }^{13} \mathrm{C} \mathrm{NMR}\left(\mathrm{CDCl}_{3}, 75 \mathrm{MHz}\right) \delta$ 23.05, 58.99 (s), 59.02 (s), 120.76 (s), 120.89 (s), 126.64, 126.66, 127.08, 127.12, 127.14, 127.26, 128.13, 128.18, 128.90, 129.74, 129.87, 134.89 (s), 134.98 (s), 135.95, 135.98, 137.33, 137.47, 138.27 (s), 138.29 (s), 146.73 (s), 146.75 (s), 178.89 (s), 179.29 (s), 197.27 (s); MS [FAB(+)] m/z (rel int) $609\left(\mathbf{M}^{+}+1,100\right)$. Anal. Calcd for $\mathrm{C}_{38} \mathrm{H}_{28} \mathrm{~N}_{2} \mathrm{O}_{2} \mathrm{~S}_{2}$ (608.78): C, 74.97; H, 4.63; N, 4.60. Found: C, 74.80; H, 4.81; N, 4.39.

Bis(3H-quinolin-4-one) 8e: yield 70\%; mp 201-202 ${ }^{\circ} \mathrm{C}$ (yellow prisms, $\mathrm{Et}_{2} \mathrm{O}$ ); IR (nujol) 1694 (vs), 1585 (vs), 1564 (vs), 1493 (m), 1283 (m), 1119 (m), 1073 (w), 1036 (w), 922 (w), 783 (m), 770 (m), 746 (m), 701 (s) $\mathrm{cm}^{-1} ;{ }^{1} \mathrm{H} \mathrm{NMR}\left(\mathrm{CDCl}_{3}, 400 \mathrm{MHz}\right) \delta$ 7.12-7.17 (m, $\left.4 \mathrm{H}\right)$, 7.30-7.31 (m, $23 \mathrm{H}), 7.50$ (dd, $1 \mathrm{H}, J=8.4,7.1 \mathrm{~Hz}), 7.60-7.63(\mathrm{~m}, 2 \mathrm{H}), 7.78-7.80(\mathrm{~m}, 2 \mathrm{H})$; ${ }^{13} \mathrm{C} \mathrm{NMR}\left(\mathrm{CDCl}_{3}, 100 \mathrm{MHz}\right) \delta 70.8$ (s), 121.8 (s), 126.6, 127.2, 127.7, 128.5, 128.6, 129.2, 130.4, 135.7, 136.2, 137.6 (s), 141.9, 146.2 (s), 177.1 (s), 196.6 (s); MS [FAB(+)] m/z (rel int) $733\left(\mathrm{M}^{+}+1\right.$, 72). Anal. Calcd for $\mathrm{C}_{48} \mathrm{H}_{32} \mathrm{~N}_{2} \mathrm{O}_{2} \mathrm{~S}_{2}$ (732.93): C, 78.66; H, 4.40; N, 3.82 . Found: C, 78.49; H, 4.31; N, 3.70.

Bis(3H-quinolin-4-one) 8f: yield 59\%; IR (nujol) 1687 (vs), 1589 (vs), 1566 (vs), 1492 (w), 1284 (m), 1219 (w), 1154 (w), 991 (m), 960 (w), 771 (m), 745 (w), 693 (m) cm ${ }^{-1}$; ${ }^{1} \mathrm{H}$ NMR $\left(\mathrm{CDCl}_{3}, 300 \mathrm{MHz}\right) \delta 2.02(\mathrm{~s}, 6 \mathrm{H}), 7.21-7.26(\mathrm{~m}, 4 \mathrm{H}), 7.28-7.33(\mathrm{~m}, 10 \mathrm{H})$, 7.44-7.52 (m, 3 $\mathrm{H}), 7.59-7.62(\mathrm{~m}, 2 \mathrm{H}), 7.20(\mathrm{t}, 1 \mathrm{H}, J=1.7 \mathrm{~Hz}), 7.86(\mathrm{dd}, 2 \mathrm{H}, J=8.3,1.6 \mathrm{~Hz}) ;{ }^{13} \mathrm{C} \mathrm{NMR}$ $\left(\mathrm{CDCl}_{3}, 75 \mathrm{MHz}\right) \delta 22.69,22.76,59.1$ (s), 120.9 (s), 126.8, 127.1, 127.4, 128.1, 128.3, 129.0, 129.2, 129.6 (s), 136.0, 136.12, 136.15, 138.22 (s), 138.24 (s), 141.9, 146.7 (s), 179.18 (s), 179.20 (s), $197.0(\mathrm{~s})$; MS $[\mathrm{FAB}(+)] \mathrm{m} / \mathrm{z}$ (rel int) $609\left(\mathrm{M}^{+}+1,100\right)$. Anal. Calcd for $\mathrm{C}_{38} \mathrm{H}_{28} \mathrm{~N}_{2} \mathrm{O}_{2} \mathrm{~S}_{2}$ (608.78): C, 74.97; H, 4.63; N, 4.60. Found: C, 74.79; H, 4.81; N, 4.47. 
Figure 1 X-Ray structure of $\mathbf{5 a}$

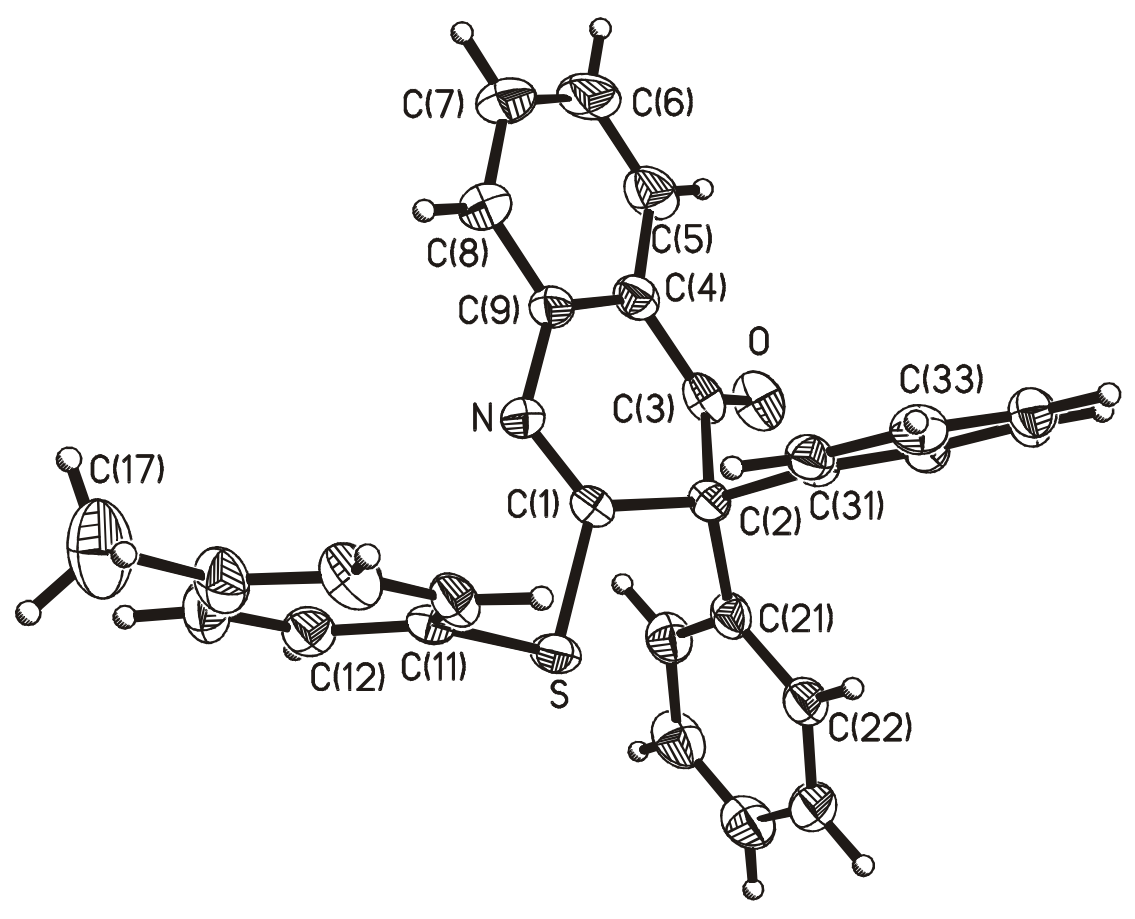


Table 1 Crystal data and structure refinement.

\begin{tabular}{|c|c|c|}
\hline Empirical formula & \multicolumn{2}{|l|}{$\mathrm{C}_{28} \mathrm{H}_{21} \mathrm{NOS}$} \\
\hline Formula weight & \multicolumn{2}{|l|}{419.52} \\
\hline Temperature & \multicolumn{2}{|l|}{ 293(2) K } \\
\hline Wavelength & \multicolumn{2}{|l|}{$0.71073 \AA$} \\
\hline Crystal system & \multicolumn{2}{|l|}{ Monoclinic } \\
\hline Space group & \multicolumn{2}{|l|}{$\mathrm{P} 2(1) / \mathrm{c}$} \\
\hline \multirow[t]{3}{*}{ Unit cell dimensions } & $\mathrm{a}=14.368(2) \AA$ & $\alpha=90^{\circ}$ \\
\hline & $\mathrm{b}=9.905(2) \AA$ & $\beta=111.17(2)^{\circ}$ \\
\hline & $c=16.546(2) \AA$ & $\gamma=90^{\circ}$ \\
\hline Volume & \multicolumn{2}{|l|}{$2195.8(6) \AA^{3}$} \\
\hline $\mathrm{Z}$ & \multicolumn{2}{|l|}{4} \\
\hline Density (calculated) & \multicolumn{2}{|l|}{$1.269 \mathrm{Mg} / \mathrm{m}^{3}$} \\
\hline Absorption coefficient & \multicolumn{2}{|l|}{$0.167 \mathrm{~mm}^{-1}$} \\
\hline $\mathrm{F}(000)$ & \multicolumn{2}{|l|}{880} \\
\hline Crystal size & \multicolumn{2}{|c|}{$0.42 \times 0.40 \times 0.20 \mathrm{~mm}^{3}$} \\
\hline Theta range for data collection & \multicolumn{2}{|l|}{3.04 to $25.00^{\circ}$} \\
\hline Index ranges & \multicolumn{2}{|c|}{$-15<=\mathrm{h}<=17,-11<=\mathrm{k}<=7,-19<=\mathrm{l}<=0$} \\
\hline Reflections collected & \multicolumn{2}{|l|}{6910} \\
\hline Independent reflections & \multicolumn{2}{|c|}{$3856[\mathrm{R}(\mathrm{int})=0.0310]$} \\
\hline Completeness to theta $=25.00^{\circ}$ & \multicolumn{2}{|l|}{$99.8 \%$} \\
\hline Refinement method & \multicolumn{2}{|c|}{ Full-matrix least-squares on $\mathrm{F}^{2}$} \\
\hline Data / restraints / parameters & \multicolumn{2}{|l|}{3856 / 24 / 281} \\
\hline Goodness-of-fit on $\mathrm{F}^{2}$ & \multicolumn{2}{|l|}{0.927} \\
\hline Final $\mathrm{R}$ indices [I>2sigma(I)] & \multicolumn{2}{|c|}{$\mathrm{R} 1=0.0367, \mathrm{wR} 2=0.0809$} \\
\hline $\mathrm{R}$ indices (all data) & \multicolumn{2}{|c|}{$\mathrm{R} 1=0.0600, \mathrm{wR} 2=0.0866$} \\
\hline Largest diff. peak and hole & 231 e $\AA^{-3}$ & \\
\hline
\end{tabular}


Table 2 Atomic coordinates $\left(\times 10^{4}\right)$ and equivalent isotropic displacement parameters $\left(\AA^{2} \times 10^{3}\right)$. U(eq) is defined as one third of the trace of the orthogonalized $\mathrm{U}_{\mathrm{ij}}$ tensor.

\begin{tabular}{|c|c|c|c|c|}
\hline & $\mathrm{x}$ & $\mathrm{y}$ & $\mathrm{z}$ & $\mathrm{U}(\mathrm{eq})$ \\
\hline S & $1547.2(3)$ & $7667.2(5)$ & $1277.8(3)$ & $29.3(1)$ \\
\hline $\mathrm{N}$ & $2370.4(10)$ & $9403.7(15)$ & $516.4(10)$ & $25.5(4)$ \\
\hline $\mathrm{O}$ & $3287.1(10)$ & $6480.1(14)$ & $-767.7(8)$ & $38.7(4)$ \\
\hline $\mathrm{C}(1)$ & 2318.7(12) & $8166.0(19)$ & $705.0(11)$ & $22.8(4)$ \\
\hline $\mathrm{C}(2)$ & 2897.3(12) & $6995.0(18)$ & $496.9(11)$ & $23.4(4)$ \\
\hline $\mathrm{C}(3)$ & $3156.0(12)$ & $7361(2)$ & $-304.9(11)$ & $28.3(4)$ \\
\hline $\mathrm{C}(4)$ & $3312.6(13)$ & $8798(2)$ & $-417.9(12)$ & $28.7(4)$ \\
\hline $\mathrm{C}(5)$ & $3815.0(15)$ & $9229(2)$ & $-953.5(13)$ & $40.6(5)$ \\
\hline$C(6)$ & $3979.9(16)$ & $10584(3)$ & $-1034.1(14)$ & $49.5(6)$ \\
\hline$C(7)$ & $3634.4(16)$ & $11520(2)$ & $-592.6(14)$ & $48.1(6)$ \\
\hline $\mathrm{C}(8)$ & $3116.5(15)$ & $11122(2)$ & $-74.8(13)$ & $37.9(5)$ \\
\hline $\mathrm{C}(9)$ & 2951.2(13) & 9758.5(19) & $18.6(12)$ & $26.9(4)$ \\
\hline $\mathrm{C}(11)$ & $1241.9(13)$ & $9246.0(18)$ & $1620.5(11)$ & 25.1(4) \\
\hline $\mathrm{C}(12)$ & $371.6(14)$ & $9918(2)$ & $1155.3(12)$ & $30.9(5)$ \\
\hline$C(13)$ & $148.5(15)$ & $11125(2)$ & $1456.4(13)$ & $39.2(5)$ \\
\hline $\mathrm{C}(14)$ & $787.9(15)$ & $11700(2)$ & $2220.7(13)$ & $38.8(5)$ \\
\hline$C(15)$ & $1644.3(15)$ & $10995(2)$ & 2691.1(13) & $38.1(5)$ \\
\hline$C(16)$ & $1869.5(13)$ & $9780(2)$ & $2401.9(12)$ & $30.4(5)$ \\
\hline $\mathrm{C}(17)$ & $566(2)$ & $13049(3)$ & $2530.9(17)$ & $67.9(8)$ \\
\hline $\mathrm{C}(21)$ & $2304.9(13)$ & $5683.2(18)$ & $383.5(12)$ & $27.6(4)$ \\
\hline $\mathrm{C}(22)$ & 2492.1(14) & $4758.5(19)$ & $1053.3(14)$ & $34.5(5)$ \\
\hline$C(23)$ & $1878.3(15)$ & $3643(2)$ & $960.1(15)$ & $42.3(6)$ \\
\hline $\mathrm{C}(24)$ & $1079.1(15)$ & $3445(2)$ & $197.5(16)$ & $45.6(6)$ \\
\hline$C(25)$ & $891.4(15)$ & $4350(2)$ & $-470.8(15)$ & $43.7(6)$ \\
\hline$C(26)$ & $1501.5(13)$ & $5462(2)$ & $-380.8(13)$ & $34.4(5)$ \\
\hline$C(31)$ & $3941.4(12)$ & $6863.7(18)$ & $1231.1(11)$ & $22.9(4)$ \\
\hline$C(32)$ & $4230.7(12)$ & 7612.4(19) & 1989.3(11) & $26.7(4)$ \\
\hline$C(33)$ & $5159.9(13)$ & $7399(2)$ & $2630.6(12)$ & $30.6(4)$ \\
\hline$C(34)$ & $5808.6(14)$ & $6454(2)$ & $2519.0(12)$ & $32.2(5)$ \\
\hline$C(35)$ & $5526.8(13)$ & $5726.4(19)$ & $1755.7(12)$ & $31.5(5)$ \\
\hline$C(36)$ & $4604.2(13)$ & 5926.3(19) & $1118.2(12)$ & $28.4(4)$ \\
\hline
\end{tabular}


Table 3 Bond lengths $[\AA]$ and angles $\left[^{\circ}\right]$.

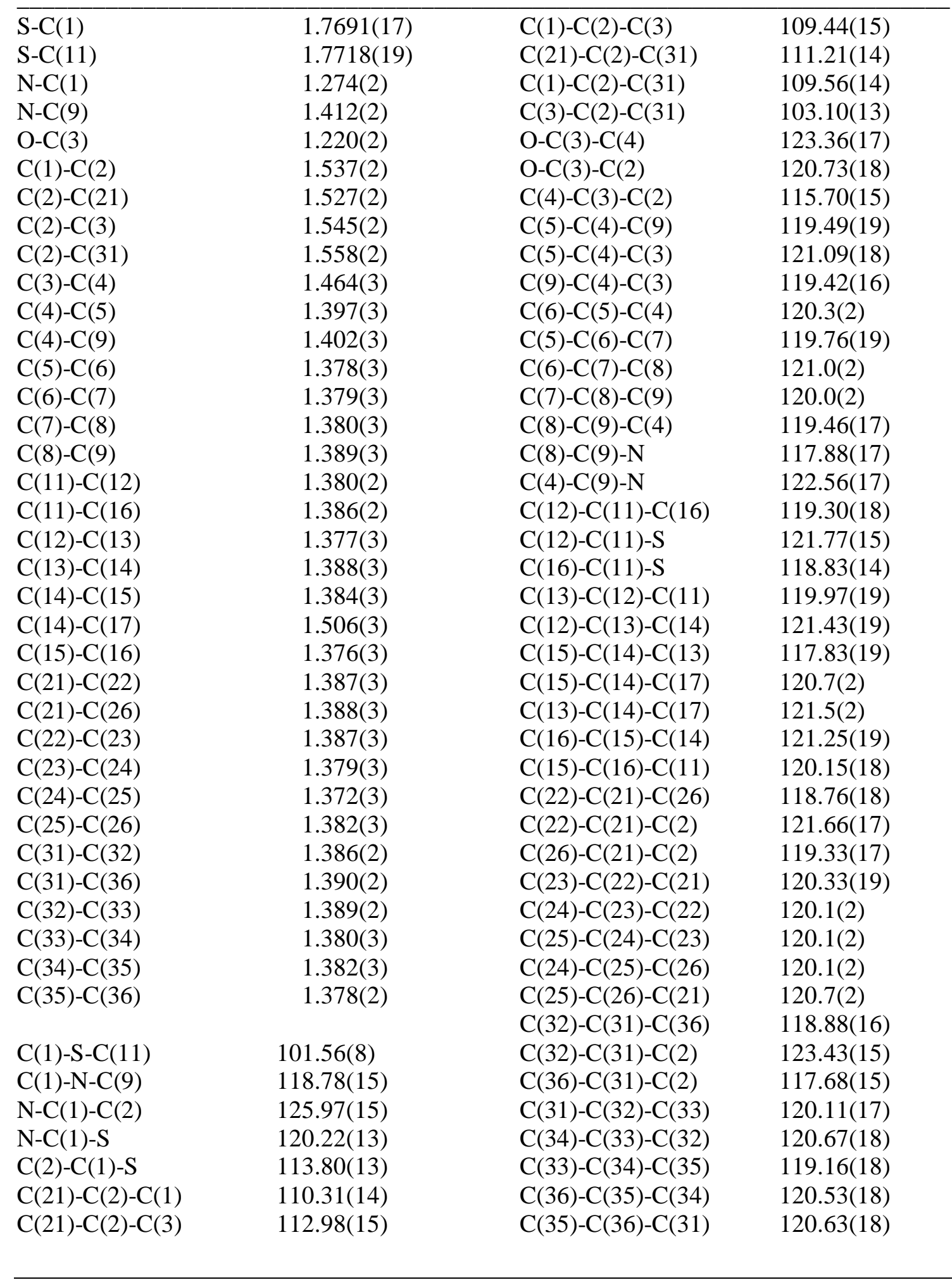


Table 4 Anisotropic displacement parameters $\left(\AA^{2} \times 10^{3}\right)$. The anisotropic displacement factor exponent takes the form: $-2 \pi^{2}\left[h^{2} a^{* 2} U^{11}+\ldots+2 h k a^{*} b^{*} U^{12}\right]$

\begin{tabular}{|c|c|c|c|c|c|c|}
\hline & $\mathrm{U}^{11}$ & $\mathrm{U}^{22}$ & $\mathrm{U}^{33}$ & $\mathrm{U}^{23}$ & $\mathrm{U}^{13}$ & $\mathrm{U}^{12}$ \\
\hline S & 29.1(2) & $27.5(3)$ & $38.0(3)$ & $0.3(2)$ & $20.1(2)$ & $-3.7(2)$ \\
\hline $\mathrm{N}$ & $25.8(8)$ & 23.7(9) & 29.9(9) & $3.8(7)$ & $13.4(7)$ & $0.6(7)$ \\
\hline $\mathrm{O}$ & $37.3(8)$ & $46.2(9)$ & $33.0(8)$ & $-14.1(7)$ & $13.2(7)$ & $2.7(7)$ \\
\hline $\mathrm{C}(1)$ & 19.0(9) & $27.7(11)$ & $22.7(9)$ & $-1.6(8)$ & $8.6(8)$ & $-1.7(8)$ \\
\hline $\mathrm{C}(2)$ & 21.3(9) & $23.5(10)$ & $26.0(10)$ & $-2.8(8)$ & $9.2(8)$ & $-0.9(8)$ \\
\hline$C(3)$ & $19.0(9)$ & 38.1(13) & $25.8(10)$ & $-5.3(9)$ & $5.9(8)$ & $2.3(9)$ \\
\hline$C(4)$ & $24.2(9)$ & $37.2(12)$ & $25.5(10)$ & $3.3(9)$ & $9.9(8)$ & $0.9(9)$ \\
\hline$C(5)$ & $36.6(12)$ & $58.1(16)$ & $33.5(12)$ & $4.9(11)$ & $20.2(10)$ & $3.7(11)$ \\
\hline$C(6)$ & $44.2(13)$ & $66.4(18)$ & $46.1(14)$ & $23.5(13)$ & $26.2(12)$ & $-3.1(12)$ \\
\hline $\mathrm{C}(7)$ & $47.4(13)$ & $44.6(15)$ & $57.0(15)$ & $19.8(12)$ & $24.6(12)$ & $-2.6(11)$ \\
\hline $\mathrm{C}(8)$ & $38.8(11)$ & $33.0(13)$ & $44.7(13)$ & $9.6(10)$ & 18.7(10) & $0.3(10)$ \\
\hline $\mathrm{C}(9)$ & $25.5(10)$ & $29.8(11)$ & $27.8(10)$ & $5.4(9)$ & $12.3(8)$ & $1.0(8)$ \\
\hline$C(11)$ & $24.6(9)$ & $29.1(11)$ & $27.0(10)$ & $3.0(8)$ & $15.9(8)$ & $-0.8(8)$ \\
\hline$C(12)$ & $31.4(10)$ & $37.8(12)$ & $24.3(10)$ & $3.7(9)$ & 11.1(9) & $0.7(9)$ \\
\hline$C(13)$ & $34.9(11)$ & $46.2(14)$ & $36.7(12)$ & $9.3(11)$ & $13.4(10)$ & $15.3(10)$ \\
\hline$C(14)$ & $43.8(12)$ & $42.8(13)$ & $36.7(12)$ & $-4.6(10)$ & $22.8(10)$ & $9.4(11)$ \\
\hline$C(15)$ & $35.9(11)$ & $50.2(14)$ & $30.0(11)$ & $-11.2(10)$ & $14.0(9)$ & $0.2(10)$ \\
\hline$C(16)$ & $20.6(9)$ & $40.6(12)$ & $29.7(11)$ & 1.1(10) & $8.8(8)$ & $2.7(9)$ \\
\hline$C(17)$ & 76.1(18) & $65.0(19)$ & $66.9(17)$ & $-15.7(15)$ & $30.9(15)$ & $24.0(15)$ \\
\hline$C(21)$ & $23.3(10)$ & $22.0(11)$ & $38.0(11)$ & $-5.3(9)$ & $11.6(9)$ & $1.1(8)$ \\
\hline $\mathrm{C}(22)$ & $25.0(10)$ & $28.0(12)$ & $49.6(13)$ & $0.5(10)$ & $12.4(9)$ & $-0.1(9)$ \\
\hline$C(23)$ & $35.2(11)$ & $26.5(12)$ & $66.2(15)$ & $6.6(11)$ & $19.3(11)$ & $1.4(9)$ \\
\hline$C(24)$ & $35.2(12)$ & $26.6(13)$ & 77.4(17) & $-14.3(12)$ & $23.2(12)$ & $-9.1(10)$ \\
\hline$C(25)$ & $29.4(11)$ & $40.4(14)$ & $56.4(15)$ & $-17.7(12)$ & $9.6(11)$ & $-8.2(10)$ \\
\hline$C(26)$ & $28.3(10)$ & $30.9(12)$ & $40.7(12)$ & $-6.5(10)$ & $8.6(9)$ & $-1.0(9)$ \\
\hline$C(31)$ & 21.2(9) & $22.5(10)$ & $26.1(10)$ & $1.1(8)$ & $9.9(8)$ & $-3.0(8)$ \\
\hline$C(32)$ & 24.2(9) & $25.5(11)$ & $31.4(10)$ & $-2.0(9)$ & $11.3(8)$ & $-2.5(8)$ \\
\hline$C(33)$ & $31.9(10)$ & $31.3(12)$ & $27.4(10)$ & $-3.8(9)$ & $9.3(8)$ & $-6.9(9)$ \\
\hline$C(34)$ & 23.3(9) & $37.9(13)$ & $31.4(11)$ & 7.1(10) & $4.8(8)$ & $-0.7(9)$ \\
\hline$C(35)$ & $25.8(10)$ & $29.6(12)$ & $39.4(12)$ & $4.6(10)$ & 12.1(9) & $3.3(9)$ \\
\hline$C(36)$ & 27.1(10) & $27.0(11)$ & $31.3(11)$ & $-2.8(9)$ & $10.8(9)$ & $-0.7(8)$ \\
\hline
\end{tabular}


Table 5 Hydrogen coordinates $\left(\times 10^{4}\right)$ and isotropic displacement parameters $\left(\AA^{2} \times 10^{3}\right)$.

\begin{tabular}{|c|c|c|c|c|}
\hline & $\mathrm{x}$ & $\mathrm{y}$ & $\mathrm{z}$ & $\mathrm{U}(\mathrm{eq})$ \\
\hline $\mathrm{H}(5)$ & 4040 & 8597 & -1257 & 49 \\
\hline $\mathrm{H}(6)$ & 4323 & 10867 & -1385 & 59 \\
\hline $\mathrm{H}(7)$ & 3752 & 12433 & -645 & 58 \\
\hline $\mathrm{H}(8)$ & 2878 & 11765 & 211 & 45 \\
\hline $\mathrm{H}(12)$ & -64 & 9556 & 639 & 37 \\
\hline $\mathrm{H}(13)$ & -443 & 11565 & 1141 & 47 \\
\hline $\mathrm{H}(15)$ & 2076 & 11349 & 3212 & 46 \\
\hline $\mathrm{H}(16)$ & 2444 & 9316 & 2731 & 36 \\
\hline $\mathrm{H}(17 \mathrm{~A})$ & -128 & 13091 & 2456 & 82 \\
\hline $\mathrm{H}(17 \mathrm{~B})$ & 718 & 13755 & 2201 & 82 \\
\hline $\mathrm{H}(17 \mathrm{C})$ & 966 & 13159 & 3133 & 82 \\
\hline $\mathrm{H}(22)$ & 3032 & 4887 & 1567 & 41 \\
\hline $\mathrm{H}(23)$ & 2006 & 3029 & 1412 & 51 \\
\hline $\mathrm{H}(24)$ & 667 & 2698 & 136 & 55 \\
\hline $\mathrm{H}(25)$ & 353 & 4214 & -985 & 52 \\
\hline $\mathrm{H}(26)$ & 1372 & 6068 & -837 & 41 \\
\hline $\mathrm{H}(32)$ & 3802 & 8259 & 2069 & 32 \\
\hline $\mathrm{H}(33)$ & 5347 & 7899 & 3140 & 37 \\
\hline $\mathrm{H}(34)$ & 6428 & 6309 & 2952 & 39 \\
\hline $\mathrm{H}(35)$ & 5963 & 5097 & 1672 & 38 \\
\hline $\mathrm{H}(36)$ & 4423 & 5429 & 608 & 34 \\
\hline
\end{tabular}


Table 6 Torsion angles $\left[{ }^{\circ}\right]$

\begin{tabular}{|c|c|c|c|}
\hline $\mathrm{C}(9)-\mathrm{N}-\mathrm{C}(1)-\mathrm{C}(2)$ & $4.7(3)$ & $\mathrm{C}(11)-\mathrm{C}(12)-\mathrm{C}(13)-\mathrm{C}(14)$ & $0.7(3)$ \\
\hline $\mathrm{C}(9)-\mathrm{N}-\mathrm{C}(1)-\mathrm{S}$ & $-176.65(13)$ & $\mathrm{C}(12)-\mathrm{C}(13)-\mathrm{C}(14)-\mathrm{C}(15)$ & $-2.4(3)$ \\
\hline $\mathrm{C}(11)-\mathrm{S}-\mathrm{C}(1)-\mathrm{N}$ & $-11.75(16)$ & $\mathrm{C}(12)-\mathrm{C}(13)-\mathrm{C}(14)-\mathrm{C}(17)$ & $177.4(2)$ \\
\hline $\mathrm{C}(11)-\mathrm{S}-\mathrm{C}(1)-\mathrm{C}(2)$ & $167.02(12)$ & $\mathrm{C}(13)-\mathrm{C}(14)-\mathrm{C}(15)-\mathrm{C}(16)$ & $1.6(3)$ \\
\hline $\mathrm{N}-\mathrm{C}(1)-\mathrm{C}(2)-\mathrm{C}(21)$ & $-151.03(17)$ & $\mathrm{C}(17)-\mathrm{C}(14)-\mathrm{C}(15)-\mathrm{C}(16)$ & $-178.1(2)$ \\
\hline S-C(1)-C(2)-C(21) & $30.28(18)$ & $\mathrm{C}(14)-\mathrm{C}(15)-\mathrm{C}(16)-\mathrm{C}(11)$ & $0.9(3)$ \\
\hline $\mathrm{N}-\mathrm{C}(1)-\mathrm{C}(2)-\mathrm{C}(3)$ & $-26.1(2)$ & $C(12)-C(11)-C(16)-C(15)$ & $-2.6(3)$ \\
\hline $\mathrm{S}-\mathrm{C}(1)-\mathrm{C}(2)-\mathrm{C}(3)$ & $155.17(12)$ & $\mathrm{S}-\mathrm{C}(11)-\mathrm{C}(16)-\mathrm{C}(15)$ & $-179.06(15)$ \\
\hline $\mathrm{N}-\mathrm{C}(1)-\mathrm{C}(2)-\mathrm{C}(31)$ & $86.2(2)$ & $\mathrm{C}(1)-\mathrm{C}(2)-\mathrm{C}(21)-\mathrm{C}(22)$ & $-97.32(19)$ \\
\hline S-C(1)-C(2)-C(31) & $-92.46(14)$ & $\mathrm{C}(3)-\mathrm{C}(2)-\mathrm{C}(21)-\mathrm{C}(22)$ & $139.83(17)$ \\
\hline $\mathrm{C}(21)-\mathrm{C}(2)-\mathrm{C}(3)-\mathrm{O}$ & $-30.8(2)$ & $C(31)-C(2)-C(21)-C(22)$ & $24.5(2)$ \\
\hline $\mathrm{C}(1)-\mathrm{C}(2)-\mathrm{C}(3)-\mathrm{O}$ & $-154.12(16)$ & $\mathrm{C}(1)-\mathrm{C}(2)-\mathrm{C}(21)-\mathrm{C}(26)$ & $76.9(2)$ \\
\hline $\mathrm{C}(31)-\mathrm{C}(2)-\mathrm{C}(3)-\mathrm{O}$ & 89.34(19) & $\mathrm{C}(3)-\mathrm{C}(2)-\mathrm{C}(21)-\mathrm{C}(26)$ & $-46.0(2)$ \\
\hline $\mathrm{C}(21)-\mathrm{C}(2)-\mathrm{C}(3)-\mathrm{C}(4)$ & $154.22(15)$ & $\mathrm{C}(31)-\mathrm{C}(2)-\mathrm{C}(21)-\mathrm{C}(26)$ & $-161.35(15)$ \\
\hline $\mathrm{C}(1)-\mathrm{C}(2)-\mathrm{C}(3)-\mathrm{C}(4)$ & $30.9(2)$ & $\mathrm{C}(26)-\mathrm{C}(21)-\mathrm{C}(22)-\mathrm{C}(23)$ & $-0.7(3)$ \\
\hline $\mathrm{C}(31)-\mathrm{C}(2)-\mathrm{C}(3)-\mathrm{C}(4)$ & $-85.64(17)$ & $\mathrm{C}(2)-\mathrm{C}(21)-\mathrm{C}(22)-\mathrm{C}(23)$ & $173.52(17)$ \\
\hline $\mathrm{O}-\mathrm{C}(3)-\mathrm{C}(4)-\mathrm{C}(5)$ & $-13.1(3)$ & $\mathrm{C}(21)-\mathrm{C}(22)-\mathrm{C}(23)-\mathrm{C}(24)$ & $0.3(3)$ \\
\hline $\mathrm{C}(2)-\mathrm{C}(3)-\mathrm{C}(4)-\mathrm{C}(5)$ & 161.71(16) & $\mathrm{C}(22)-\mathrm{C}(23)-\mathrm{C}(24)-\mathrm{C}(25)$ & $0.1(3)$ \\
\hline $\mathrm{O}-\mathrm{C}(3)-\mathrm{C}(4)-\mathrm{C}(9)$ & $166.81(17)$ & $\mathrm{C}(23)-\mathrm{C}(24)-\mathrm{C}(25)-\mathrm{C}(26)$ & $-0.1(3)$ \\
\hline $\mathrm{C}(2)-\mathrm{C}(3)-\mathrm{C}(4)-\mathrm{C}(9)$ & $-18.3(2)$ & $\mathrm{C}(24)-\mathrm{C}(25)-\mathrm{C}(26)-\mathrm{C}(21)$ & $-0.4(3)$ \\
\hline $\mathrm{C}(9)-\mathrm{C}(4)-\mathrm{C}(5)-\mathrm{C}(6)$ & $1.8(3)$ & $\mathrm{C}(22)-\mathrm{C}(21)-\mathrm{C}(26)-\mathrm{C}(25)$ & $0.8(3)$ \\
\hline $\mathrm{C}(3)-\mathrm{C}(4)-\mathrm{C}(5)-\mathrm{C}(6)$ & $-178.23(18)$ & $\mathrm{C}(2)-\mathrm{C}(21)-\mathrm{C}(26)-\mathrm{C}(25)$ & $-173.60(17)$ \\
\hline $\mathrm{C}(4)-\mathrm{C}(5)-\mathrm{C}(6)-\mathrm{C}(7)$ & $-0.9(3)$ & $C(21)-C(2)-C(31)-C(32)$ & $-115.83(18)$ \\
\hline$C(5)-C(6)-C(7)-C(8)$ & $-0.6(3)$ & $\mathrm{C}(1)-\mathrm{C}(2)-\mathrm{C}(31)-\mathrm{C}(32)$ & $6.4(2)$ \\
\hline $\mathrm{C}(6)-\mathrm{C}(7)-\mathrm{C}(8)-\mathrm{C}(9)$ & $1.1(3)$ & $\mathrm{C}(3)-\mathrm{C}(2)-\mathrm{C}(31)-\mathrm{C}(32)$ & $122.82(18)$ \\
\hline $\mathrm{C}(7)-\mathrm{C}(8)-\mathrm{C}(9)-\mathrm{C}(4)$ & $-0.1(3)$ & $\mathrm{C}(21)-\mathrm{C}(2)-\mathrm{C}(31)-\mathrm{C}(36)$ & $63.0(2)$ \\
\hline $\mathrm{C}(7)-\mathrm{C}(8)-\mathrm{C}(9)-\mathrm{N}$ & $-176.57(18)$ & $\mathrm{C}(1)-\mathrm{C}(2)-\mathrm{C}(31)-\mathrm{C}(36)$ & $-174.76(15)$ \\
\hline $\mathrm{C}(5)-\mathrm{C}(4)-\mathrm{C}(9)-\mathrm{C}(8)$ & $-1.3(3)$ & $\mathrm{C}(3)-\mathrm{C}(2)-\mathrm{C}(31)-\mathrm{C}(36)$ & $-58.3(2)$ \\
\hline $\mathrm{C}(3)-\mathrm{C}(4)-\mathrm{C}(9)-\mathrm{C}(8)$ & $178.75(17)$ & $\mathrm{C}(36)-\mathrm{C}(31)-\mathrm{C}(32)-\mathrm{C}(33)$ & $-1.5(3)$ \\
\hline $\mathrm{C}(5)-\mathrm{C}(4)-\mathrm{C}(9)-\mathrm{N}$ & $174.97(17)$ & $\mathrm{C}(2)-\mathrm{C}(31)-\mathrm{C}(32)-\mathrm{C}(33)$ & $177.34(16)$ \\
\hline $\mathrm{C}(3)-\mathrm{C}(4)-\mathrm{C}(9)-\mathrm{N}$ & $-5.0(3)$ & $\mathrm{C}(31)-\mathrm{C}(32)-\mathrm{C}(33)-\mathrm{C}(34)$ & $0.6(3)$ \\
\hline $\mathrm{C}(1)-\mathrm{N}-\mathrm{C}(9)-\mathrm{C}(8)$ & $-170.90(17)$ & $\mathrm{C}(32)-\mathrm{C}(33)-\mathrm{C}(34)-\mathrm{C}(35)$ & $0.6(3)$ \\
\hline $\mathrm{C}(1)-\mathrm{N}-\mathrm{C}(9)-\mathrm{C}(4)$ & $12.8(3)$ & $\mathrm{C}(33)-\mathrm{C}(34)-\mathrm{C}(35)-\mathrm{C}(36)$ & $-0.9(3)$ \\
\hline $\mathrm{C}(1)-\mathrm{S}-\mathrm{C}(11)-\mathrm{C}(12)$ & $95.35(15)$ & $\mathrm{C}(34)-\mathrm{C}(35)-\mathrm{C}(36)-\mathrm{C}(31)$ & $0.1(3)$ \\
\hline C(1)-S-C(11)-C(16) & $-88.27(15)$ & $\mathrm{C}(32)-\mathrm{C}(31)-\mathrm{C}(36)-\mathrm{C}(35)$ & $1.2(3)$ \\
\hline$C(16)-C(11)-C(12)-C(13)$ & $1.8(3)$ & $\mathrm{C}(2)-\mathrm{C}(31)-\mathrm{C}(36)-\mathrm{C}(35)$ & $-177.75(16)$ \\
\hline S-C(11)-C(12)-C(13) & $178.18(14)$ & & \\
\hline
\end{tabular}


Table 7 Hydrogen bonds [ $\AA$ and ${ }^{\circ}$ ].

\begin{tabular}{lcccc}
\hline D-H...A & d(D-H) & d(H...A $)$ & $d(D \ldots A)$ & $<($ DHA $)$ \\
\hline C(16)-H(16)...\#1 & 0.93 & 2.47 & $3.228(2)$ & 139.2 \\
\hline
\end{tabular}

Symmetry transformations used to generate equivalent atoms: \#1 $\mathrm{x},-\mathrm{y}+3 / 2, \mathrm{z}+1 / 2$

Cartesian coordinates of the stationary points found in the conversion $\mathbf{1 1} \rightarrow \mathbf{1 2} \rightarrow \mathbf{1 3}$ optimized at he B3LYP/6-31+G* theoretical level.
11
C,-1.4437412776,2.0320700209,0.1047052217
C, $-2.6570363761,2.126972854,0.59648298$
$\mathrm{N},-0.2929954859,2.0323511165,-0.3036505354$
C, $0.4358969648,1.2522047831,-1.1713637996$
C, $0.8440542435,-0.0260830087,-0.9808782481$
C, $0.485744158,-0.8646936275,0.1692195463$
S, $1.3490711124,-2.4704230026,0.1371336917$
$\mathrm{H},-2.9395148722,1.5101079977,1.443611407$
H,-3.3882972024,2.7939616539,0.1523008323
$\mathrm{H}, 0.7965722509,1.7984428084,-2.0411514761$
$\mathrm{H}, 1.512948125,-0.4510969028,-1.7230269843$
O,- $-0.2837308858,-0.570042243,1.0652880025$
$\mathrm{H}, 0.7644631122,-2.9135935176,1.2683826808$

\section{TS1}

C, $1.169083648,-0.8089185849,-0.8947983192$

C, $2.0856246034,-1.7548001469,-0.7050452241$

$\mathrm{N}, 0.5060259426,-0.1363324076,-1.7372129298$

C,-0.6518806198,0.5200626334,-1.6864297166

C,-1.4188298866,0.8795294159,-0.5880984512

C,-1.1849217391,0.6094225265,0.7902579993

S, $0.6364356851,0.1050550734,1.2276635923$

$\mathrm{H}, 2.4741885075,-2.0082305755,0.2705821411$

$\mathrm{H}, 2.4562659332,-2.2867890779,-1.5778230806$

$\mathrm{H},-1.0268536681,0.8219182926,-2.6623751514$

$\mathrm{H},-2.3546185589,1.3932939725,-0.7870815772$

O,-1.939966213,0.705927761,1.7283689976

H, $0.2511388958,-0.9659440844,1.9523009899$ 
12

C,-0.1453358176,0.2974970246,-0.9792874806

C, $-0.3363066659,-0.95202931,-1.4415690741$

$\mathrm{N}, 1.1030067122,0.8454574143,-0.6745853834$

C, $1.7566376305,0.4402376977,0.3552104375$

C, $1.4059335776,-0.536319806,1.3781614289$

C, $0.3165289668,-1.3038220003,1.4730637147$

S, $-1.5260978007,1.4250426577,-0.7581767118$

H,-1.3158718558,-1.2987967729,-1.7528185234

$\mathrm{H}, 0.502994169,-1.6322382711,-1.5526277444$

$\mathrm{H}, 2.7312227248,0.9034433826,0.515389402$

H, 2.0918935677,-0.6579731652,2.2136003606

$\mathrm{O},-0.5645685355,-2.0314941854,1.6984679496$

H,-0.7819186434, 2.5452522518,-0.8718361781

\section{TS2}

C, $0.3058318726,0.8915695817,-0.0618284466$

C,0.7129710096,0.2459781854,-1.1953861711

$\mathrm{N}, 0.8015571809,0.6023290566,1.189608203$

C,0.776251423,-0.6504847028, 1.5476435106

C, $0.2642426925,-1.780854478,0.8269405127$

C, $-0.1175913795,-1.8104458893,-0.4767360418$

S,-0.9593793077,2.135093436,-0.1388130426

$\mathrm{H}, 0.2766851606,0.4678064017,-2.1641391607$

$\mathrm{H}, 1.6518129805,-0.300208999,-1.1913851016$

H,1.1628154066,-0.8646162217,2.5440790787

$\mathrm{H}, 0.0924575053,-2.7076603606,1.3694296984$

O,-0.5006089458,-2.3258633419,-1.4632302918

H,-1.0899645371,2.2592113621,1.1978088974

\section{3}

C,0.164279171,-0.8058331452,0.0252788529

C,-0.1071098854,0.2768838665,-0.9935783888

$\mathrm{N}, 0.1953397486,-0.6338212768,1.3018602151$

C,-0.0397715495,0.6545713823,1.7936297202

C,-0.3013794414,1.7591582375,1.0510133911

C, $-0.3580528697,1.6689805164,-0.4032109947$

S, $0.4543431591,-2.4141830072,-0.6531119764$

$\mathrm{H}, 0.7348494565,0.3486842139,-1.6955778308$

H,-0.9759549613,0.0028609102,-1.6074715465

$\mathrm{H}, 0.0039794364,0.714604543,2.8783147822$

$\mathrm{H},-0.4733151541,2.7267318382,1.5117434264$

O,-0.5888630564,2.6198439339,-1.1416596233

H,0.6366843374,-3.0105210696,0.5442427888 


\section{References}

1 2-Azidobenzoyl chloride 1a and 2-azido-5-chlorobenzoyl chloride $\mathbf{1 b}$ were both prepared following the experimental procedure described for 1a: Porter, T. C.; Smalley, R. K.; Teguiche, M.; Purwono, B. Synthesis 1997, 773.

2 Crich, D.; Yao, Q. J. Org. Chem. 1996, 61, 3566.

3 Stratmann, O.; Kaiser, B.; Fröhlich, R.; Meyer, O.; Hoppe, D. Chem. Eur. J. 2001, 7, 423.

4 Taylor, E. C.; McKillop, A.; Hawks, G. H. Org. Synth. 1973, 52, 36.

5 Pracejus, H.; Wallura, G. J. Prakt. Chem. 1962, 19, 33. 